\title{
Abstracts of the 4th Symposium on Neuropsychological Rehabilitation 9-10 July 2007, San Sebastián (Donostia), Spain
}

\section{A Satellite Symposium of the Midyear Meeting of the International Neuropsychological Society, 4-7 July, Bilbao, Spain}

\section{Organising Committee:}

Barbara A. Wilson (Convenor), MRC Cognition and Brain Sciences Unit, Cambridge and The Oliver Zangwill Centre, Ely, United Kingdom

Michael Perdices (Program Chair), Department of Neurology, Royal North Shore Hospital, Sydney, Australia

Jonathan Evans, University of Glasgow, United Kingdom

Sara Fernández Guinea, Department of Basic Psychology (Cognitive Processes), Faculty of Psychology. Universidad Complutense of Madrid, Madrid, Spain

Gerhard Mueller, Akademie bei König \& Müller, Würzburg, Germany

Nuria Paúl, Hospital Beata Maria Ana, Unidad de Daño Cerebral, Madrid, Spain, Departamento de Psiquiatría y Psicología Médica. Facultad de Medicina, Universidad Complutense de Madrid, Spain

Ignacio Quemada, Brain Damage Service, Aita Menni Hospital. Bilbao. Spain

Robyn Tate, Rehabilitation Studies Unit, Northern Clinical School, Faculty of Medicine, University of Sydney, and Royal Rehabilitation Centre Sydney, Australia

\section{SESSION 1: THE BIG PICTURE}

\section{Formation of the Special Interest Group in Neuropsychological Rehabilitation}

B.A. Wilson

MRC Cognition and Brain Sciences Unit, Cambridge and The Oliver Zangwill Centre, Ely, United Kingdom

$\mathrm{T}$ he inaugural meeting of the New Special Interest Group in Neuropsychological Rehabilitation takes place in San Sebastian, Spain. The origins of this group are described and reasons for setting it up under the auspices of the World Federation of Neurological Rehabilitation (WFNR) are discussed. An historical overview is provided including the embryonic lunchtime discussion in an Australian winery which led to a decision to hold a satellite meeting of interested parties following on from the INS midyear conference to be held in Brisbane in 2004. This first meeting was organised by Robyn Tate and was held in Uluru (Ayers Rock) in July of that year. Because of its success, a decision was made to hold a post INS satellite meeting annually. At the second meeting, held in Galway (2005), it was decided that a group of interested individuals should consider inaugurating a new international society comprising (primarily but not exclusively) neuropsychologists, occupational therapists and speech and language therapists. To this end, I was asked to investigate 
the possibility of such a society and report back at the third meeting to be held in Liechtenstein. After considerable debate, it was decided to join forces with the WFNR and in July 2006 the new Special Interest Group was formed. This was announced at the Liechtenstein meeting and we decided to hold the first international meeting of this group in Spain 2007. We expect to hold many more meetings in the years to come.

\title{
A Practical Clinical Trial of Holistic Neuropsychological Rehabilitation
}

\author{
K.D. Cicerone, T. Mott, J. Azulay, M. Sharlow-Galella, W. Ellmo, J. Friel and S. Paradise \\ JFK - Johnson Rehabilitation Institute, Edison, New Jersey, United States of America
}

\begin{abstract}
$\mathrm{D}$ escriptive studies of comprehensive-holistic neuropsychological rehabilitation suggest clinical benefits on neuropsychological functioning and community integration after traumatic brain injury (TBI), although randomised controlled trials in this area have shown equivocal or negative results. We conducted a prospective, randomised controlled trial comparing intensive-holistic neuropsychological rehabilitation (INP) and 'standard' neurorehabilitation (STD) after TBI. Participants were recruited from clinical and community referrals seeking postacute brain injury rehabilitation. Sixty-eight participants were randomly allocated to receive 4 months of INP $(n=34)$ or STD $(n=34)$. Prior to treatment groups were equivalent on demographic variables, neuropsychological functioning (NP), community integration (CIQ) and perceived life satisfaction (PQOL). INP consisted of individual and group interventions emphasising self-regulation, interpersonal communication, and functional problem solving. STD consisted of physical therapy, occupational therapy, speech therapy and neuropsychological treatment. Results were evaluated using intent-to-treat analyses with repeated measures ANOVAs. Both groups improved their NP functioning $(p<.001)$ with no interaction effect. There was no main effect of treatment on CIQ $(p=.14)$ with a significant treatment interaction effect $(p=.015)$ : INP demonstrated significant improvement $(p=.004)$ while STD did not change. There was a significant overall effect of treatment $(p=.011)$ and treatment interaction effect $(p=.049)$ on PQOL due to the improvement of the INP group $(p=.004)$ with no benefit related to STD treatment. Conclusions: There are significant benefits of intensive-holistic rehabilitation on community integration and life satisfaction compared with standard rehabilitation after TBI.
\end{abstract}

\section{Under what Conditions is an Impairment Focused or Restitution Approach Appropriate in Neuropsychological Rehabilitation?}

\section{J.J. Evans}

University of Glasgow, United Kingdom

ne of the great debates within neuropsychological rehabilitation centres around the question of whether treatment interventions should focus at the level of cognitive impairments or at the level of disabilities/activity limitations. Furthermore, if the focus is on impairments, should the aim be to restore normal cognitive processing or to compensate for impairment through the use of some form of cognitive, behavioural or environmental strategy? The answers to these questions are crucial to determining what happens in rehabilitation settings, but the ongoing nature of the debate reflects the fact that the answers are not yet 
clear or at least not yet agreed. In this paper I will review the evidence relevant to addressing these questions in relation to the cognitive domains of attention, executive functioning, language and memory. I will suggest that it is possible to determine some general principles governing the conditions under which an impairment/restitution based approach is appropriate and conclude that there are at least 3 conditions under which an impairment focused approach might be effective: (1) where the specific content of cognitive training is the thing that needs to be acquired, (2) where increased top-down, conscious verbal control is likely to benefit task performance, and (3) where activation of one cognitive system has the effect of stimulating or inhibiting an impaired system. The question of which, if any, of these approaches might be considered to be 'restorative' is also examined.

\title{
Neuropsychological Rehabilitation: What is it All About?
}

S. Sopeña, ${ }^{1}$ R. Rous, ${ }^{2}$ and B.A. Wilson ${ }^{1,2}$

${ }^{1}$ MRC Cognition and Brain Sciences Unit, Cambridge, United Kingdom

${ }^{2}$ The Oliver Zangwill Centre for Neuropsychological Rehabilitation, Ely, United Kingdom

\begin{abstract}
Qjectives: Clinical neuropsychologists have traditionally concerned themselves with the assessment and remediation of cognitive deficits resulting from brain injury, little is known, however, about the psychological theories and models that inform clinical practice in this field. Wilson's (2002) model of cognitive rehabilitation suggests that rehabilitation is one of many fields that need a broad theoretical base incorporating frameworks, theories and models from different areas. If rehabilitation is constrained by one model this can lead to important aspects of patients' lives being neglected. This study represents the first attempt to reveal the variety and combination of approaches used by neuropsychologists in the United Kingdom (UK) today. Methods: An interview was carried out either face-to-face or by telephone with 54 neuropsychologists actively engaged in brain injury rehabilitation in the UK. Questions were related to which of the components of Wilson's (2002) model were being used by practicing clinicians in the field. Results: All participants reported employing standardised and behavioural measures to assess cognitive, emotional and psychosocial consequences of brain injury. A goal planning approach to rehabilitation was followed by $100 \%$ of participants. Fifty-seven different models/theories were cited by clinicians as influencing their clinical practice, with CBT being the most frequently used. CT scans were the most commonly neuroimaging technique used to aid in the understanding of the injury. Conclusions: The study showed that clinical neuropsychologists in the UK use a range of theoretical approaches to their work. Clinical psychology models seem to have an increased influence on the practice of neuropsychological rehabilitation.
\end{abstract}

\section{It's Just One Brain: Integrating Cognitive, Emotional, and Eispositional Interventions}

C. Mateer

University of Victoria, Canada

Strategies to improve or manage cognitive impairments too often ignore the impact of emotions and beliefs on generalisation of skills and 
changes in adaptive functioning. This paper will describe research findings on the critical interplay of cognition, emotion, and metacognitive beliefs. It will describe approaches to and the value of integrating, within interventions, activities designed to increase awareness, overcome disuse of cognitive skills, and enhance cognitive self-efficacy.

\title{
SESSION 2: BEYOND COGNITION
}

\section{Replicated Positive Results for the Vocational Case Coordinator (VCC) Model of Vocational Intervention After Acquired Brain Injury (ABI) Within the Social Model of Disability}

J.F. Malec, ${ }^{1}$ and A. M. Moessner ${ }^{2}$

${ }^{1}$ Department of Psychiatry and Psychology, Mayo Clinic College of Medicine, Rochester, Minnesota, United States of America

${ }^{2}$ Department of Nursing, Mayo Clinic College of Medicine, Rochester, Minnesota, United States of America

\begin{abstract}
$B^{\prime}$ ackground: Prior research indicates the rate of long term communitybased employment (CBE) after acquired brain injury (ABI) is below 30-40\%. In contrast, studies of specialised ABI vocational services report long-term CBE rates exceeding 60\%-70\%. Purpose: To attempt to replicate results of our original study of the Vocational Case Coordinator (VCC) Model of vocational intervention after ABI in which $81 \%$ of 114 participants maintained $\mathrm{CBE}$ for at least 1 year after initial placement $(53 \%$ of total sample with no support). Design: Referral cohort studied within the social model of disability. In contrast to the medical model, which targets change in the individual only, the social model also works to minimise social and attitudinal barriers, promote more positive attitudes and social access, and develop accommodations for cognitive and behavioural disabilities. Participants: 138 people with ABI entered the vocational program an average of 3.5 years postinjury. Interventions: VCC assisted participants to develop self-directed vocational plans and networks of medical centre and community services. Early intervention, work trials, temporary or long-term supported employment, and employer education are fundamental features of the VCC Model. Main Findings: $80 \%$ of participants sustained CBE for at least 1 year after initial placement (56\% of the total sample with no support). As in the prior study, better outcomes were associated with earlier intervention and more circumscribed disability but not with initial injury severity. Conclusions: Results provide additional evidence of the effectiveness of the VCC model. Implications for social change within the social model of disability will be discussed.
\end{abstract}

\section{Understanding Inappropriate Social Behaviour After Brain Injury: Contributions of Social Cognition}

I. Sánchez-Cubillo, ${ }^{1}$ and J.I. Quemada ${ }^{2}$

'Brain Damage Service, Aita Menni Hospital, Bilbao, Spain; and Psychology Department, University of Balearic Islands, Palma de Mallorca, Spain

2 Brain Damage Service, Aita Menni Hospital, Bilbao, Spain

Tntroduction: Disinhibited or socially inappropriate behaviour is one of the 1 most common disorders after brain injury. Recent studies suggest the usefulness of the neuropsychology of social cognition in the explanation of its 
etiopathology. Theory of Mind (ToM), emotion recognition, and emotion awareness may play a role in the production of these behaviours. Additionally, growing evidence suggests that these processes may dissociate from executive functions. Objective: The aims of this study are: (1) to explore the explanatory capability of social cognition processes in the disinhibited behaviour, and (2) to test the correlation/dissociation of executive functions and social cognition functioning. A single-case methodology is used, reporting a patient with persistent inappropriate social behaviour. A neuropsychological assessment was conducted, including evaluation of social cognition processes, such as ToM and facial expression recognition. Executive functions and decision making are also assessed. Results: The patient performed correctly on tests of executive functions and decisionmaking, but failed on the tasks assessing social cognition processes. These results are discussed under the focus of the latest theoretical approaches. A working framework for understanding, assessing and treating some disinhibited patients is proposed. Conclusions: Behavioural disinhibition seems, at least partially, influenced by ToM, emotion recognition and emotion awareness. The dissociation between these processes and executive functions is supported by these results. Working with social cognition paradigms in neuropsychological rehabilitation looks a promising area.

\title{
Reconnecting With Society: Social Identity Continuity and the Importance of Maintained Group Memberships for Wellbeing After Stroke
}

\author{
C. Haslam, A. Holme and S.A. Haslam \\ School of Psychology, University of Exeter, Washington Singer Laboratories, Exeter, United Kingdom
}

\begin{abstract}
$A^{\prime}$ ims: A growing literature in acquired brain injury shows that negative life transitions pose a major threat to adjustment and wellbeing which can compromise rehabilitation. Relevant to this issue is social psychological research showing that group memberships - and the sense of social identity associated with them - are a basis for social support that can buffer individuals from the negative consequences of stressful life events. Our study is the first to explore the relationships between multiple and maintained group memberships and wellbeing in adjustment to stroke. Methods: 53 people who had suffered stroke completed (a) the The Exeter Identity Transition Scale (EXITS) assessing group membership before and after stroke, (b) the Cognitive Failures Questionnaire, and (c) measures of wellbeing. Results: As predicted on the basis of a social identity approach to mental health, life satisfaction was associated with multiple group memberships prior to stroke and with the maintenance of group memberships. The importance of identity continuity to wellbeing was also suggested by path analysis. This indicated that belonging to multiple groups is associated with enhanced wellbeing due to the greater likelihood that group memberships will be preserved in the context of a significant life transition. It also indicated that cognitive failures compromise wellbeing because they make it harder for people to maintain those same group memberships. Results support the view that social identity processes and mental health are interdependent, and that sensitivity to this point in the context of neuropsychological rehabilitation can have theoretical and practical benefits.
\end{abstract}




\title{
Long Term Functional Outcome From Low Awareness States
}

\author{
B.K. Dewar, ${ }^{1}$ M.R. Coleman, ${ }^{2}$ J.D. Pickard ${ }^{3}$ and B.A. Wilson ${ }^{4}$ \\ ${ }^{1}$ Institute of Psychiatry, St Thomas' Hospital, London, United Kingdom \\ ${ }_{2}^{2}$ Division of Academic Neurology, Addenbrookes Hospital, Cambridge, United Kingdom \\ ${ }^{3}$ Wolfson Brain Imaging Centre, University of Cambridge, Cambridge, United Kingdom \\ ${ }^{4}$ Medical Research Council Cognition and Brain Sciences Unit, Cambridge, United Kingdom
}

\begin{abstract}
Deople in a low awareness state may be in coma, a vegetative state (VS) or $P$ in a minimally conscious state (MCS). There have been only a few studies investigating the long-term functional outcome of people in low awareness states. We report an exploratory study into the functional outcome at 2 years of people in low awareness states. Thirteen brain-injured patients considered to meet the diagnostic criteria for the VS and MCS were recruited into a retrospective follow-up study, on average 33 months postictus. All patients were assessed on the Wessex Head Injury Matrix (WHIM; Shiel, Wilson, McLellan, Horn, \& Watson, 2000), and measures of disability, motor function and a psychosocial questionnaire. The mean time between ictus and initial WHIM as reported in this study was 28 months. On the basis of clinical investigation at the time of initial WHIM, 6 of the subjects were deemed to be in VS and 7 in MCS. At follow-up, improvement was seen in 11 of the 13 subjects, with a higher number of total behaviours recorded on the WHIM. Nine of the 13 subjects recorded more advanced behaviours on the WHIM at follow-up. However, our sample had a high level of functional dependence and poor motor function. Results will be discussed in terms of the rehabilitation needs for people in low awareness states.
\end{abstract}

\section{Explaining the Inexplicable - Intensity of Attention and Fatigue in Multiple Sclerosis}

H. König, ${ }^{1}$ J. Volkert, ${ }^{2}$ G. W. Alpers ${ }^{2}$ and P. Flachenecker ${ }^{3,4}$

${ }^{1}$ Centre of Clinical Neuropsychology, Würzburg, German.

${ }^{2}$ Department of Biological Psychology, Clinical Psychology, and Psychotherapy, University of Würzburg, Germany

${ }^{3}$ Department of Neurology, University of Würzburg, German.

${ }^{4}$ Neurological Rehabilitation Centre Quellenhof, Bad Wildbad, Germany

Gatigue is one of the most common and most disabling symptoms in patients with multiple sclerosis (MS). Yet, up to now, it is poorly defined and understood. Most previous investigations did not find correlations of subjectively experienced fatigue with cognitive functions measured with neuropsychological tests. We suggest that these attempts failed for 2 reasons. First, methods used to measure fatigue usually did not meet psychometric criteria. Second, previous research typically focussed on quality (i.e., errors) rather than on intensity (i.e., reaction time) of attentional performance. We, therefore, developed a more specific self-report questionnaire with excellent psychometric properties (Würzburg Fatigue Inventory for Multiple Sclerosis) and tested intensity of attention. In a study with 57 MS patients we aimed to objectively measure the attentional consequences of fatigue. Patients participated in an extended test session with several attention tests from a computerised assessment battery (TAP). It could be shown that patients' self-reported level of fatigue significantly correlated with several measures of intensity of attention (alertness, sustained attention). On the other hand, selectivity measures did not show any relationship to fatigue. These findings contribute to a better understanding of fatigue. 


\section{Managing Risk in Rehabilitation}

A. Worthington

Brain Injury Rehabilitation Trust, Birmingham, United Kingdom

R ehabilitation is, inherently, a risky enterprise. By promoting indepenRdence with persons who may have poor judgment and impulsive behaviour, clinicians have to balance multiple competing interests. Risk management is becoming increasingly central to brain injury rehabilitation, especially for people with a significant cognitive and behaviour disorder. However, practice is largely athoeretical and methods are borrowed from other areas such as mental health. The emphasis of clinical risk assessment on reducing error and the resulting safety first approach is contrary to the spirit of rehabilitation, but reflects the changing attitudes to risk in society at large. Elsewhere, the traditional actuarial approach to risk is now replaced by cognitive and behavioural models, which have yet to influence clinical practice. It is argued that research on decision making from neuroeconomics can be combined with psychological models of risk to form the basis of a new approach to risk management that is more in keeping with the realities of rehabilitation. This thesis is explored with preliminary data from healthy and brain-injured populations.

\section{SESSION 3: MEASUREMENT AND EVIDENCE}

\section{Validation of a Cognitive Assessment as a Predictor of Fitness to Drive in People With Dementia}

N.B. Lincoln, K. Vella and R. Smith

University of Nottingham, United Kingdom

Rackground: Cognitive tests (e.g., MMSE) may be used to advice

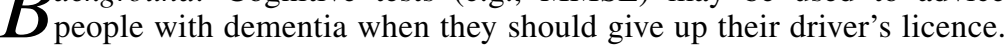
Some tests have been validated with 'on road' assessments for people with dementia. Linclon et al., (2006) previously developed a battery of tests, which correctly classified $92 \%$ of participants with dementia as safe or unsafe to drive on the basis of performance on 6 tests. Aim: To examine the predictive validity of cognitive tests as indicators of fitness to drive in an independent sample of people with dementia. Methods: People with dementia ( $n=25 ; 19$ males) aged 60 to 89 years (mean 75.8, SD 7.7), were assessed on a battery of cognitive tests, including measures of memory, concentration, reasoning and perceptual abilities, and a prediction made about their performance on the road. Sixteen were assessed on the road by a driving instructor, blind to the cognitive test prediction. Participants were graded as safe or unsafe to drive. Results: Performance on the road was cross-tabulated with the predictive equation. Using a cut off score of 5 to identify safe drivers, the equation correctly identified 7 out of 8 patients who were definitely safe to drive. When a cut-off score of 0 was used one person who was definitely safe to drive was misclassified. Conclusion: Validation of the predictive equation will indicate whether the screening assessment may be used to identify patients with dementia who are likely to be safe to drive and others who require further assessment. 


\title{
Development of a Questionnaire to Assess Experience of Divided Attention Difficulties: Insights Gained from Rasch Analysis
}

\author{
A. Bateman, ${ }^{1}$ E. Greenfield, ${ }^{1}$ J. Evans ${ }^{2}$ and B.A.Wilson ${ }^{1,3}$ \\ ${ }^{1}$ The Oliver Zangwill Centre, Ely, United Kingdom \\ 2 University of Glasgow, United Kingdom \\ ${ }^{3}$ MRC Cognition and Brain Sciences Unit, Cambridge, United Kingdom
}

\begin{abstract}
fter a brain injury (BI) many people have a problem with dual tasking A(DT), this is thought to impact on many aspects of activities of daily living, returning to work and leisure. A new battery of tests of divided attention and DT ('The DivA') measures performance on cognitive and motor tasks in isolation and combined. Also included in the battery is a 24-item questionnaire that provides an indication of the frequency of day-to-day activity restrictions that may be typical consequences of impairments in divided attention. This presentation describes the item response characteristics in an analysis of 100 brain-injured patient's responses to the self-rating questionnaire. A partial-credit Rasch model was used to evaluate the data using commercially available software (RUMM2020, Andrich 2004). A person separation index of $a=0.94$ indicates that 5 separate groups of patients can be identified using this measure. Five items displayed disordered thresholds indicating the need for rescoring. One item displayed significant $(P<.001)$ misfit from the model indicating the need to remove this item. Principal components analysis of residuals provided evidence of 2 subscales, labelled activities and impairments. After rescoring to reduce the number of thresholds, and arrangement of the data into these 2 subscales, the resulting analysis demonstrated good fit to the Rasch model (activity scale chi square $22.17, p=.56$; impairment scale $40.5, p=.44$ ). We conclude that the questionnaire provides a reliable, robust and valid measurement of the construct. This analysis provides a platform for further research in divided attention using this questionnaire.
\end{abstract}

\section{Measuring Social Communication Ability After Traumatic Brain Injury: Reliability and Validity of the La Trobe Communication Questionnaire}

\author{
J.M. Douglas, C.A. Bracy and P.C. Snow \\ La Trobe University, Victoria, Australia
}

Self- and close other-reports of ability can provide a valuable and timeSefficient means of clinical assessment during rehabilitation. The $\mathrm{La}$ Trobe Communication Questionnaire (LCQ) measures social communication ability from multiple perspectives. It is a 30-item questionnaire based on universal principles underlying social discourse. Both English and Spanish versions are available. The current research was undertaken to evaluate the internal consistency, test-retest reliability and validity for self-report and close other versions of the tool when used with brain-injured adults. Participants were 88 adults with severe traumatic brain injury (TBI) and 71 close others (relatives or friends). Internal consistency coefficients were above 0.9 and test-retest reliability exceeded 0.80 . The questionnaire was sensitive to the effect of severity of injury. Principal component factor analysis with Varimax rotation revealed a stable factor structure that reflects the pathophysiology of TBI and the complex interplay between cognitive and communication processes that social discourse demands. Results support the use of the LCQ as a reliable measure of social communication ability in adults with severe TBI. It can be used to evaluate specific behaviours as well 
as dimensions associated with breakdown in conversational interaction (inhibitory control, conversational fluency, attentional control and task management). In addition, comparison of self and close other scores can be used to evaluate a client's awareness of deficits specific to the domain of social communication.

\title{
Reliability and Validity of a New Scale for Rating Methodological Quality of Single-Subject Designs and N-of-1 Trials
}

\author{
R. Tate, ${ }^{1,2}$ S. McDonald, ${ }^{3}$ M. Perdices,${ }^{4}$ L. Togher, ${ }^{5}$ R. Schultz, ${ }^{1}$ and S. Savage ${ }^{1}$ \\ ${ }^{1}$ Rehabilitation Studies Unit, Northern Clinical School, Faculty of Medicine, University of Sydney, \\ Australia \\ ${ }^{2}$ Royal Rehabilitation Centre Sydney, Australia \\ ${ }^{3}$ School of Psychology, University of New South Wales, Sydney, Australia \\ ${ }^{4}$ Department of Neurology, Royal North Shore Hospital, Sydney, Australia \\ ${ }^{5}$ School of Communication Sciences and Disorders, Faculty of Health Sciences, University of Sydney, \\ Australia
}

\begin{abstract}
Background: Scales are currently available to rate the methodological $\boldsymbol{B}_{\text {quality of randomised and nonrandomised controlled trials, but there are }}$ none that assess single-subject designs (SSDs). The objective of this study was to develop a methodological rating scale for SSDs and evaluate its reliability. Method: The Single-Case Experimental Design Scale (SCEDS) was developed and refined over a 3-year period. It consists of 11 items, with 10 items assessing methodological quality. Content validity was addressed by identifying items to reduce the main sources of bias in SSDs as stipulated by authorities in the field. It was empirically tested against 85 published reports using SSD methodology archived in PsycBITE ${ }^{\mathrm{TM}}$ (www.psycbite.com), the electronic database of all published studies on the nonpharmacological treatments for neurological conditions. Six clinical researchers, trained and experienced in rating methodological quality of clinical trials, developed the scale and participated in reliability studies. Inter-rater reliability was assessed using a random sample of 20/312 SSD reports. Results: Inter-rater reliability for the total score was excellent, both for individual raters (overall $\mathrm{ICC}=0.83$; range $0.77-0.91$ ) and for consensus ratings between pairs of raters (overall $\mathrm{ICC}=0.88$; range $\mathrm{ICC}=0.84-0.91$ ). Item reliability was fair to excellent for consensus ratings between pairs of raters (range $k=0.48$ to 1.00). Conclusions: The SCEDS provides a brief and valid evaluation of methodological quality of SSDs, with the total score demonstrating excellent inter-rater reliability. Items from the scale can also be used as a checklist in the design, reporting and critical appraisal of SSDs, thereby assisting to improve standards of SSD methodology.
\end{abstract}

\section{Single Case Designs in Rehabilitation: How Do We Interpret the Results?}

\author{
M. Perdices ${ }^{1}$ and R.L.Tate ${ }^{2}$ \\ ${ }^{1}$ Department of Neurology, Royal North Shore Hospital, Sydney, Australia \\ 2 Rehabilitation Studies Unit, Northern Clinical School, Faculty of Medicine, University of Sydney, \\ and Royal Rehabilitation Centre Sydney, Australia
}

The aim of this paper is to critically appraise strengths and weaknesses of the methods used for interpreting results of single-subject designs (SSDs). The majority (40\%) of published studies examining the effects of nonpharmacological interventions for rehabilitation of psychological consequences of acquired brain impairment are SSDs. Well-designed SSDs 
have several advantages over randomised-control trials (RCTs): (a) the applicability of treatment affects to individual patients is more easily determined from SSDs than RCTs; (b) SSDs provide a means to evaluate interventions for rarely occurring deficits (eg., prosopagnosia) where RCTs may not be plausible; (c) SSDs have smaller time/resource 'costs' than RCTs; and (d) SSDs can be readily integrated with clinical practice. In essence, SSDs are fundamental tools for determining efficacy of treatment in the rehabilitation setting. But, how can you tell if the treatment has worked? There are 2 main approaches to this question: visual versus statistical analysis of results. There are no formal criteria or decision rules governing visual analysis, inter-rater agreement is low and there is relatively poor agreement between visual and statistical analysis of data. On the other hand, visual analysis emphasises clinical rather than statistical significance. The distinction and pertinence of these 2 aspects of significance is discussed. The statistical approach allows for control of extraneous factors and is useful when baseline data is not stable, or the treatment effect not well understood. Several standard statistical tests for SSDs are reviewed.

\section{SESSION 4: PAEDIATRIC PERSPECTIVES}

\section{Sustained Favorable Effects of Cognitive Training in Children With Acquired Brain Injuries}

I. van 't Hooft,' K. Andersson, ${ }^{1}$ B. Bergman, ${ }^{4}$ T. Sejersen, ${ }^{1}$ L. von Wendt ${ }^{2}$ and A. Bartfai ${ }^{3}$

${ }^{7}$ Neuropaediatric Unit, Astrid Lindgren's Children's Hospital, Karolinska University Hospital, Stockholm, Sweden

${ }^{2}$ Department of Child Neurology, University Central Hospital, Helsinki, Finland.

${ }^{3}$ Department of Rehabilitation Medicine, Karolinska Institute, Danderyds Hospital, Stockholm, Sweden

${ }^{4}$ Children's University Hospital, Lund, Sweden

The overall aim of the present study was to assess in greater detail the sus1 tained effects of a broad-based cognitive training program on the neuropsychological performance of children with acquired brain injury. In particular, the long-term (6 months) effects on cognitive functions, as well as how various moderators (gender, age at the time of injury/diagnosis, time since injury/ diagnosis, age at the training) might influence outcome were investigated. A group of 38 children, 9-16 years of age, with various types of acquired brain injury had earlier been randomly assigned into treatment and control groups. These 2 groups had first been assessed directly after completion of the training and were now reassessed 6 months later. The treatment group exhibited significantly more persistent improvements with respect to complex tasks of attention and memory in comparison to the control group. In contrast, there were no differences on simple reaction time tests. We conclude that the long-term effects on cognitive functions of this broad-based neurocognitive training is encouraging. These positive results should be further investigated in larger groups and in different settings. 


\title{
Parents of Children With Brain Injury: Stress, Coping and Parenting Strategies
}

W.H. Williams ${ }^{1}$, K.C. Chua, ${ }^{2}$ L. Hooper ${ }^{3}$ and I. Frampton ${ }^{1}$

${ }^{1}$ Centre for Clinical Neuropsychology Research (CCNR), School of Psychology University of Exeter, United Kingdom

${ }^{2}$ Ministry of Community Development, Youth \& Sports, Republic of Singapore

${ }^{3}$ School of Health Professions - Peninsula Allied Health Centre, College of St Mark and St John Plymouth, Devon, United Kingdom

\begin{abstract}
Caring for a survivor of Acquired Brain Injury (ABI) is, potentially, stressful. It is often noted that parents of children with brain injury, as 'significant others', may be protected from some of the stresses of caring. That is, compared to a partner of an adult survivor, a parent is already 'in role' as provider of guidance, support and care. However, not only is parenting, in itself, not without its stresses, the sequelae of brain injury are very likely to be difficult for a parent to manage, and could lead to stress. Caregiver stress interferes with the parent's psychological adjustment, and can compromise their capacity to support the child. We describe 2 studies in which we addressed the issues of coping, parenting and stress. In study one, with 52 parents of children with ABI, we explored coping and parenting style as correlates of caregiver stress. Hierarchical linear regression analyses suggested that coping and parenting strategies reliably predicted caregiver depression and anxiety. Posthoc probing of moderator effects further suggested that presence of dysexecutive disorder may be particularly related to parental distress. In study 2 we examined the specific impact of childhood encephalitis, and whether the illness was 'recent' or 'remote' (within or beyond 7 years of 'now') with 36 parents of children aged between 10 and 17. Neurobehavioural consequences of childhood encephalitis appear to persist over time and, again, higher levels of dysexecutive symptoms were associated with greater parental distress. Implications of these studies, regarding support for parents, are discussed.
\end{abstract}

\section{Socioemotional Functioning in Young Adolescents With a History of TBI: Developing a New Measure}

\begin{abstract}
S.E. Wall, W.H. Williams and I. Frampton
Centre for Clinical Neuropsychology Research (CCNR), School of Psychology, University of Exeter, United Kingdom
\end{abstract}

ims: Despite the prevalence of social and emotional difficulties reported
in children and adolescents with a history of acquired brain injury (ABI),
there is a lack of suitable assessment measures. Furthermore, individuals
often have poor insight into their impairments, causing conflicts within the
family as well as complicating the process of intervention. Consequently, the
aim of this study was to develop a measure of social-emotional function and
insight for children and young adolescents with a history of ABI. Method:
The Socio-Emotional Questionnaire for Children (SEQ-C) was completed
by 2510 - to 15 -year-olds with a history of ABI and 50 gender and age-
matched controls, and their parent/guardian. Results: The total scores for
both the self- and parent-report versions of the SEQ-C were lower for the
ABI sample than the controls, indicating more efficient functioning in the
control group. Reliable differences were observed on the parent-report sub-
scales according to the age at injury (pre- or post-7 years) and location of
damage (frontal or no-frontal). On the self-report, scores on the Emotion
Recognition and Empathy subscale varied according to reported injury 
severity. Greater discrepancy between the parent and child totals was seen in the ABI sample than the controls, and they tended to rate themselves as more efficient. The discrepancy scores varied according to injury type (acquired or traumatic) and location. Implications: The SEQ-C has the potential to be used to highlight impairments in socio-emotional functioning and insight, and thus inform the focus and design of intervention strategies.

\section{The Forgotten Siblings}

H. Harrington

Epworth Healthcare/Bouverie, La Trobe University, Australia

Tntroduction: Siblings form a unique relationship with each other that dif1 fers from any other relationship formed. It is expected if a young adult's life is changed by suffering a traumatic brain injury (TBI) their sibling's life is also affected. Studies and clinical practice has tended to focus upon the emotional responses of the primary care giver. This is most often not the sibling. The ongoing impact upon the young adult sibling's life following TBI is relatively unknown. Aims: This paper aims to present the findings of this insightful study of the siblings' experience and provide recommendations for professionals to assist the sibling. Method: This qualitative study aimed to explore this topic. Four siblings of young TBI adult participated in an in-depth interview reflecting upon how this event impacted their life. The principles of grounded theory were applied to the study and a thematic analysis of the interviews was completed. Results: The findings of the study identified that these siblings were significantly impacted for many years following the TBI. Each sibling described an ongoing mixture of strong emotional responses. They felt profound sadness for their losses and for their sibling's losses. They constantly worried for their injured sibling's future. Love and admiration of their sibling's achievements were also highlighted. Each sibling described changed relationships with family and friends. However, their lives displayed positive adaptation and growth despite these complex changes. The siblings in this study felt neglected and forgotten by the professionals.

\section{SESSION 5: EXECUTIVE DOMAINS}

\section{Cognitive Rehabilitation of the Dysexecutive Syndrome: Effects of a Multifaceted Treatment Program on Neuropsychological Tests for EF and Indications of Everyday Participation Level Functioning}

J.M. Spikman, D. Boelen, K.F. Lamberts, W.H. Brouwer and L. Fasotti

Department of Neurology, University Medical Centre Groningen, Postbus, Netherlands

$\mathrm{M}$ any patients who suffered a stroke or a TBI have dysexecutive problems. This Dysexecutive Syndrome (DES) forms a major impediment to successful re-entry to the community, affecting quality of life of both patients and relatives negatively. Effective rehabilitation methods aiming at treatment of the whole range of symptoms are sorely needed. Existent treatment programs usually address only a single aspect of the DES, which has limited clinical relevance and ecological validity. For this reason, we developed a 'multifaceted treatment of the dysexecutive syndrome', Patients learn to cope with a broad range of goal setting, planning and regulation problems, 
with the final aim to improve everyday executive functioning (EF). The treatment exists of a protocol in which skills and strategies are taught that can be individually tailored to the patient's particular problems, needs and goals. The protocol is comprised of 3 modules, namely: (1) Information and Awareness, (2) Goal setting and Planning, and (3) Initiative, Regulation and Problem Solving. For every subject, treatment consists of 241 -hour sessions divided over a period of 3 months. In an RCT, in which 10 Dutch rehabilitation centres took part, the affectivity of this treatment was assessed. Eighty patients with executive problems were randomly assigned to either the experimental treatment or to a control treatment (computerised training for improving general cognitive functioning). The effectiveness of the experimental treatment was assessed with neuropsychological EF tests, rating scales and questionnaires measuring EF in everyday participation level activities as well as the attainment of pre-set goals.

\title{
Cognitive Rehabilitation of Brain-Injured Patients With the Dysexecutive Syndrome: Functional Brain Reorganisation Assessed With the Aid of fMRI
}

\author{
K.F. Lamberts, ${ }^{1,2,3}$ R.J. Renken, ${ }^{3}$ J.M. Spikman ${ }^{1}$ and W.H. Brouwer ${ }^{1}$ \\ ${ }^{1}$ Department of Neurology, University Medical Centre Groningen, the Netherlands \\ ${ }^{2}$ Revalidatie Friesland, the Netherlands \\ ${ }^{3} \mathrm{BCN}$ - Neuroimaging Centre Groningen, the Netherlands
}

The dysexecutive syndrome leads to difficulties in functioning independently and resuming previous activities, thus affecting the quality of life of patients and their relatives. Because of the high prevalence of dysexecutive problems after brain injury and their negative influence on rehabilitation in general, effective treatment is needed. This study is a supplement to a larger multicentre research project: treatment of the dysexecutive syndrome. The objective is to determine whether and how a multifaceted executive function treatment improves the everyday problem solving and planning capabilities of patients with the dysexecutive syndrome. Within the same time schedule of evaluating neuropsychological test performance and 'quality of life', patients are presented with executive function tasks in the fMRI scanner: before treatment, right after ending, and 6 months after treatment. We expect to find an increase of prefrontal cerebral blood flow and this to be the strongest right after ending the treatment. Following treatment we expect patients to resume previous activities and thus create a more challenging environment for themselves resulting in a more routine like handling of every day planning activities. Consequently, less effort should be visible during the performance of executive tasks. The wide variability in anatomical and functional organisation after brain injury implies the use of a singlesubject design. We will present patient data and make a comparison with our healthy control group. By combining neuroimaging data with neuropsychological test and 'quality of life' data, the results are more informative than when each set of data would be considered separately. 


\title{
The Leading Role of Executive Functioning in the Functional Independence of Acquired Brain Injury Patients
}

I. Bombín, ${ }^{1}$ A. Bilbao ${ }^{2}$ and T. Legido ${ }^{3}$

${ }^{1}$ Reintegra: Centro de Rehabilitación Integral. Oviedo, Spain

${ }^{2}$ Centro Estatal de Atención al Daño Cerebral. Madrid, Spain

${ }^{3}$ Hospital Dr Rodríguez Lafora, Madrid, Spain

\begin{abstract}
Clinical consequences of acquired brain injury (ABI) include cognitive, emotional, and behavioural impairment that compromise the patients' functional independence. However, the differential weight among the main cognitive domains in predicting long-term functional outcome and independence has not been yet well established. Seventy-eight patients with acquired brain injury (ABI) were assessed at admission and discharge to a comprehensive rehabilitation program by means of a neuropsychological battery that included measures of overall cognitive functioning, attention, memory, working memory, and executive functioning. Six months after discharge, functional independence was measured by the Craig Handicap Assessment and Reporting Technique (CHART). Additionally, qualitative information regarding the type and degree of functional occupation (paid job, voluntary work, housekeeping) was gathered. Pearson correlations between cognitive variables and the CHART were performed to look for associations. In order to look for differential explanatory weights, lineal regression analyses were performed, with cognitive measures as independent variables and CHART values as dependent variables. A multinomial logistic regression analysis was also performed to determine which cognitive variables best predicted the type and degree of occupation postdischarge. All cognitive domains, both at admission and discharge, predicted functional independence postdischarge (Pearson $r=0.386-0.864)$. However, when the linear regression analyses were performed, executive functioning at discharge was the best predictor of functional independence $\left(R^{2}=0.467-0.751\right)$. Additionally, executive functioning was the best predictor of independent occupation postdischarge. Our results confer to executive functioning a leading role in functional independence achievement. These results will be discussed in the context of experimental factor models of executive functions.
\end{abstract}

\section{Clinical Implications of Categorical Cueing During Verbal and Nonverbal Learning in Persons With Unilateral Frontal and Temporal Lobe Tumours}

\author{
M. Maitz ${ }^{1}$ and M. Perdices ${ }^{2}$ \\ ${ }^{7}$ School of Psychology, University of Sydney, New South Wales, Australia \\ ${ }^{2}$ Department of Neurology, Royal North Shore Hospital, St Leonards, New South Wales, Australia
}

$\boldsymbol{B}_{\text {ackground: This study explored the clinical significance of providing }}$ $\boldsymbol{B}_{\text {categorical cues at different stages of learning in persons with unilateral }}$ frontal and temporal lobe tumours. We examined whether frequency of clinical memory impairment (FCMI) was normalised after the provision of cues. Method: Participants were 58 patients (left frontal $=17$, right frontal $=20$, left temporal $=10$, right temporal $=11$ ), and 20 controls. Verbal and nonverbal 'list learning' tasks were administered and cues provided at either (a) encoding stage only, (b) retrieval stage only, (c) both encoding/retrieval stages, and (d) no stage (baseline). Scores " 2 standard deviations below the control group's mean baseline performance were classified as clinically impaired. FCMI at cueing and baseline conditions were compared. Results: Cueing at either encoding or retrieval on the verbal task 
reduced FCMI to within normal limits for all performance measures in both Frontal groups. Cueing at both encoding/retrieval reduced FCMI to within normal limits for all performance measures in the Right Temporal group, and for recall consistency and long delay recall in the Left Temporal group. Cueing at either encoding or retrieval on the nonverbal task reduced FCMI to within normal limits only in the left frontal group for recall consistency. Conclusion: Categorical cueing produces clinically significant improvement of memory deficits, albeit cueing differentially affects specific facets of memory. Our findings also challenge well established notions of modalityspecific lateralisation of memory functions, and highlight the importance of developing interventions for the rehabilitation of memory that are empirically based and tailored to address specific deficits of individual patients.

\title{
The Rehabilitation of Executive Deficits Following Traumatic Brain Injury
}

\author{
J. Ponsford, ${ }^{1,2,3}$ M.K. Grant ${ }^{1}$ and P.Bennett ${ }^{1}$ \\ ${ }^{1}$ School of Psychology, Psychiatry, and Psychological Medicine, Monash University, Melbourne, Australia \\ 2 Monash-Epworth Rehabilitation Research Centre, Epworth Hospital, Melbourne, Australia \\ ${ }^{3}$ National Trauma Research Institute, Melbourne, Australia
}

Z xecutive dysfunction is characterised by disorganised behaviour and plan- ning difficulties. To date, few rehabilitation studies have specifically targeted executive dysfunction. One exception is a study by Levine et al. (2000) which used Goal Management Training (GMT) to improve meal preparation performance in an individual with executive difficulties. GMT is a structured rehabilitation protocol that uses a hierarchy of 5 steps to train the individual in various aspects of goal attainment, including attention, problem definition, problem solving, and monitoring. The purpose of the present study was to investigate the clinical validity of GMT in the remediation of executive deficits in individuals with traumatic brain injury, and to determine whether any gains imparted through GMT generalised to nontargeted goal-directed activities. A multiple case study design was used, applying GMT to an activity with which the participant was experiencing planning/organisational difficulties. Outcomes were assessed using Goal Attainment Scaling. This paper will present the results for several case studies, the focus of which was on reducing the amount of money the participant was spending on weekly household shopping. For all cases the primary goal was achieved. However, less success was achieved with secondary goals imposed by the therapist. Significant improvements were also recorded between baseline and the postintervention measurement phases on the Multiple Errands Task, providing some evidence for the generalisation of training benefits to nontargeted activities.

\section{SESSION 6: REHABILITATION OF MEMORY}

\section{You Learn Something New Every Day - Or Do You?}

\author{
E. Greenfield, R. Nannery and B.A. Wilson \\ Medical Research Council, Cognition \& Brain Sciences Unit, Cambridge, United Kingdom
}

The ability to learn and remember how to perform new, multistage tasks is essential to developing and maintaining essential life skills, whether learning to use an electronic memory aid or the correct sequence for personal care activities. It is generally accepted that even some densely amnesic 
individuals retain intact procedural learning, even if there is no recollection of the process of learning. Our aim was to develop a novel task that would assess the ability to learn new information. It would be a valuable addition to the Rivermead Behavioural Memory Test (RBMT) that was being updated. The criteria for the test were: to be original, to have 6 stages, to be manageable with either motor or language problems, to be compact, low cost and have 2 parallel forms. We chose a 6-piece 'Star/Square' assembly task, based on a geometric theorem. There were 3 learning trials and one delayed. We looked at 80 neurologically impaired people with TBI, stroke, encephalitis or dementia, (some with severe memory problems), and how they compared with healthy controls. The subjects also completed the new version of the RBMT and other neuropsychological tests. The controls all learned the test within the 3 trials, but many of the neurologically impaired group showed impairment related to the severity of their condition and the aetiology. It is a quick, reliable test to indicate whether people with memory problems still have the capacity to learn, for example, how to use a memory aid.

\title{
Prospective Memory Rehabilitation for Individuals With Traumatic Brain Injury: A Randomised Controlled Trial
}

\author{
D. Shum, ${ }^{1}$ J. Fleming, ${ }^{2}$ H. Gill ${ }^{3}$ and N. Roche ${ }^{4}$ \\ ${ }^{1}$ School of Psychology and Applied Cognitive Neuroscience Research Centre, Griffith University, Australia \\ ${ }^{2}$ Division of Occupational Therapy, University of Queensland, Australia \\ ${ }^{3}$ Applied Cognitive Neuroscience Research Centre, Griffith University, Queensland, Australia \\ ${ }^{4}$ Princess Alexandra Hospital, Brisbane, Australia
}

Prospective memory (PM) involves the delayed execution of an intended
action. Impairments of PM have implications for everyday living and
independent functioning. As such, this type of memory is of great clinical
and theoretical importance. Individuals with traumatic brain injury (TBI), as
well as other neuropsychological patients, commonly experience problems
with prospective memory. Some examples of PM problems include failure to
attend an appointment, take required medication, or switch off electrical
appliances before leaving the house. In this paper we report results of a ran-
domised controlled trial designed to treat prospective memory problems using
self-awareness and compensatory training. The purpose of the study was to
test new treatment approaches in the field of prospective memory rehabilita-
tion. Forty participants with moderate-to-severe TBI were allocated to 1 of 4
treatment conditions. Each participant received 2 sessions of self-awareness or
placebo training followed by 6 sessions of remedial or compensatory training.
Data were collected by an assessor blind to the treatment condition at prein-
tervention, postintervention and 6 month follow-up assessments. Measures
included psychometric tests of prospective memory, quantity and quality of
diary use, self-report and significant others' report on frequency of prospective
memory lapses, and ratings of community integration. Results suggested that
the self-awareness plus compensatory training group showed significant treat-
ment gains on a range of variables at postintervention. 


\title{
Improvement of Memory Performance in Elderly People: Benefits of Semantic Categorisation Strategy Training
}

S. Fernández Guinea and M. L. Delgado Losada

Department of Basic Psychology (Cognitive Processes), Faculty of Psychology, Universidad Complutense of Madrid, Spain

\begin{abstract}
Trtroduction: The aim of the study was to analyze the long-term benefits of a memory training program. An ecological semantic categorisation strategy training was applied to a sample of people older than 60 years with subjective memory complains but without neurological or psychiatric disease. Training focused on the abilities to: (a) abstract common semantic features of words and objects, (b) group elements in semantic categories; (c) denominate categories; (d) remember categories' names; and (e) remember elements of each category. Method: 130 elderly subjects were divided randomly in 2 groups of 65 people (experimental and control). A Spanish adaptation of California Verbal Learning Test was administered to all subjects before and after training and 6 months later. Results: In the posttraining evaluation there were significant statistical differences between groups in the following variables: total amount of words learned in 5 trials ( $F$ $=46,565 ; p<.001)$, semantic strategies $(F=81,804 ; p<.001)$, short-term recall $(F=15,082 ; p<.001)$, short-term recall with semantic clues $(F=15,706 ; p<.001)$, long-term recall $(F=8,149 ; p<.05)$, and long-term recall with semantic clues $(F=10,073 ; p<.05)$. The experimental group got better results than control group in all measures. These benefits maintained in the 6 months follow-up evaluation in the following variables: total amount of words learned in 5 trials $(F=40,815 ; p<.001)$, semantic strategies $(F=17,658 ; p<.001)$ and short-term recall $(F=7,157 ; p<.05)$. Conclusion: Semantic categorisation strategy training was useful for elderly people with subjective memory complaints. Benefits were maintained for at least 6 months after training because elderly people keep using this technique in their daily living activities.
\end{abstract}

\section{A Comparison of the Effectiveness of Two Types of Memory Rehabilitation Strategies Following Brain Damage: A Single Blind, Randomised Control Trial}

R. das Nair, N. Lincoln, E. Kontou, K. Vella and M.C. O'Brien

Institute of Work, Health, and Organisations, University of Nottingham, United Kingdom

$\mathrm{M}$ emory difficulties are common following traumatic brain injury (TBI), stroke, or multiple sclerosis (MS). There is inconclusive evidence of the effectiveness of memory rehabilitation strategies due to a paucity of well conducted randomised controlled trails (RCTs). Cautiously positive results have been reported with single-case designs and small group studies. Objective: To compare the effectiveness of a 'compensation-based' (use of internal and external memory aids) and a 'restitution-based' (use of internal memory aids) memory rehabilitation program, with a self-help control group using a single-blind RCT. Methods: Participants with memory problems were recruited. Memory functions, mood, and activities of daily living were assessed at baseline and follow-up. Participants were randomly allocated (in blocks of 4) to one of the 3 programs by an independent agency. The rehabilitation comprised 10 weekly sessions. Follow-up assessments were carried out 5 and 7 months after randomisation by an assessor, blind to group allocation. Independent assessors also recorded the group process using 
time-sampling. Results: 36 participants were randomised and a split-plot ANOVA used to analyse the data. There was no significant main effect of time or group $(p>.05)$ on the Rivermead Behavioural Memory Test or the General Health Questionnaire, but a significant main effect of time was observed on the Everyday Memory Questionnaire $(p=.04)$. Conclusions: There was no evidence of effectiveness of memory rehabilitation. However, the small sample size and lack of sensitive assessment tools may have affected outcomes. Furthermore, large discrepancies between subjective reports and objective tests of functioning were observed at follow-up.

\title{
Errorless Learning and Spaced Retrieval: Who Benefits From These Techniques?
}

\author{
K. I. Hodder, ${ }^{1}$ C. Haslam ${ }^{1}$ and P.J. Yates ${ }^{1,2}$ \\ ${ }^{1}$ School of Psychology, University of Exeter, Washington Singer Laboratories, United Kingdom \\ ${ }_{2}^{2}$ Mardon Neurorehabilitation Centre, Exeter, United Kingdom
}

\begin{abstract}
$A$ ims: Two particularly promising techniques used in memory rehabilitation are errorless learning and spaced retrieval. However, their effectiveness has rarely been compared and not in a sufficiently controlled manner to draw meaningful conclusions. Furthermore, these techniques may prove effective for different people, and little evidence exists as to why this might be. This research directly compared these techniques under controlled conditions, and investigated which patients benefited from the techniques. Methods: Thirty patients with acquired brain injury learnt face-name associations under errorless learning, spaced retrieval and trial-and-error conditions. Measures of executive functioning, attention and stage of memory impairment were then used in an attempt to relate these functions to the benefit gained from each technique. Results: Spaced retrieval proved to be the most effective technique; however this was not the case for every patient. Measures of executive functioning and attention provided little evidence that these domains were related to the degree of benefit gained from the techniques. Finally, measures of encoding, storage and retrieval were obtained for each patient. The advantage of errorless learning was not related to these measures; however, the spaced retrieval advantage correlated with a measure of retrieval ability, such that those with poorer retrieval skills benefited more from spaced retrieval. This research highlights the need to identify which patients benefit from different memory rehabilitation techniques so that effective methods can be targeted for each individual.
\end{abstract}

\section{SESSION 7: PUSHING THE BOUNDARIES WITH TECHNOLOGY \\ Flexing SenseCam's Muscle: Long-Term Memory Reconsolidation in a Severely Amnesic Encephalitic Patient}

\author{
A.S. Jansari, C. Stadtler, E. Berry and G. Browne \\ School of Psychology, University of East London, United Kingdom
}

$\mathrm{W}$ ithin the last decade, a growing literature has shown that it is possible to use external memory aids such as NeuroPage for alleviating some of the prospective memory problems experienced by brain-damaged individuals (Wilson, 2001). However, given the importance of autobiographical memory in maintaining a sense of self, improving the ability to remember past long-term memories could have a significant psychosocial impact on an individual's wellbeing. Berry et al. (2007, in press) have shown how it is 
possible to use a novel device, known as SenseCam to improve long-term recall in a memory-impaired limbic encephalitis patient whose memory faded within a week; they suggest that SenseCam aids in visual reconsolidation of weak rapidly fading memory traces. We report the case of another encephalitic who is so severely amnesic that her memory is functionally nonexistent 5 minutes after an event; we address the extent to which SenseCam could have beneficial effects in a patient at the most severe end of the amnesic spectrum. Using a reconsolidation paradigm, we were able to show that with SenseCam NP is able to recall events for much longer periods than she has previously. The implications for the use of SenseCam with patients of differing extents of memory-impairment are discussed.

\title{
Virtual Reality Exploration of Social Rule Violation in Patients With Focal Prefrontal Neurosurgical Lesions
}

\author{
R.G. Morris, ${ }^{1}$ E. Pullen, ${ }^{2}$ S Kerr, ${ }^{3}$ P.R. Bullock ${ }^{4}$ and R.P. Selway ${ }^{4}$ \\ ${ }^{1}$ Neuropsychology Unit, Department of Psychology, Institute of Psychiatry, London, United Kingdom \\ ${ }^{2}$ Department of Psychology, Institute of Psychiatry, London, United Kingdom \\ ${ }^{3}$ VIRART, Human Factors Research Group, Innovative Technology Research Centre, School M3, \\ University of Nottingham, United Kingdom \\ ${ }^{4}$ Department of Neurosurgery, King's College Hospital, London, United Kingdom
}

\begin{abstract}
$\mathrm{V}$ irtual reality (VR) was used to explore social cognition in 22 prefrontal neurosurgical lesions and 22 normal controls. The study used a virtual reality social environment consisting of a bar in which drinks could be ordered and groups of people in the bar. The participants were required to walked from the far end of the bar to the counter, order drinks and return their starting place. They had the choice moving between other people (socially inappropriate) or around the people (social appropriate). They were distracted during their movement by subsidiary tasks in order to make the procedure more sensitive to social dysfunction. The patients were split into those with neurosurgical lesions impinging on the either the orbitofrontal (OF), dorsolateral (DL) or mesial (M) region of the prefrontal cortex. The OF group showed a significant increase in social inappropriate behaviour in comparison to the controls. The study shows that VR can be used to simulate social functioning in brain damaged patients and may provide a rapid method of measuring social ability.
\end{abstract}

\section{Using SenseCam, a Wearable Camera, to Alleviate Autobiographical Memory Loss}

G. Browne, ${ }^{1.2}$ E. Berry ${ }^{1,2}$ and N. Kapur ${ }^{1}$

${ }^{\prime}$ Addenbrooke's Hospital, Cambridge, United Kingdom

${ }^{2}$ Sensors and Devices Group, Microsoft Research, Cambridge, United Kingdom

$\mathrm{W}$ e describe some preliminary results in which patients in the early stages of Alzheimer's disease showed improvements in their autobiographical memories after viewing images taken by SenseCam, a small wearable camera. We have already established the effectiveness of this camera as an autobiographical memory aid in a patient with severe memory impairment following limbic encephalitis, and have described associated improvements to psychological wellbeing. We aimed to establish whether SenseCam could similarly benefit patients with Alzheimer's disease. SenseCam has been developed by Microsoft Research, in Cambridge, in the United Kingdom. Worn around the neck, it uses sensors to take photographs automatically in 
response to environmental changes and records several hundred images during the course of a day. The images can be uploaded to a PC and viewed as a rapid slide show. In the current study we used SenseCam as a pictorial diary to cue and consolidate autobiographical memories of significant events in 3 patients with Alzheimer's disease. Preliminary results showed significant benefits to memory and psychological wellbeing following review of the images over a 2 -week period. We propose that systematically viewing SenseCam images helps in the consolidation and retrieval of memories that would otherwise be lost. We describe how patients and their carers report many benefits of using this technology, including joy of sharing experiences again, and improved confidence in the knowledge that autobiographical memories can be easily retrieved and enjoyed.

\title{
A Novel Assistive Technology for Cognition to Aid Performance of Complex Behavioural Sequences
}

\author{
A. Gillespie ${ }^{1}$ and B. O'Neill ${ }^{2}$ \\ ${ }^{1}$ University of Stirling, United Kingdom \\ 2 West of Scotland Mobility and Rehabilitation Centre, Glasgow, United Kingdom
}

Introduction: A variety of brain pathologies can result in difficulties performing behavioural sequences. Complex sequences underpin many activities of daily living and therefore sequencing deficits are severely disabling. Interventions for executive function are therefore largely social (increasing support) or behavioural (environmental modification and training). Assistive technologies for cognition have the potential to revolutionise care of those with dysexecutive difficulties. Prospective memory systems have demonstrated some of this promise. Previous assistive technologies for cognition have limitations in terms of cognitive demands and interface. We propose a novel system for assisting executive function, the GUIDE $^{\odot}$. Participants: Patients with vascular cognitive impairment undergoing physical rehabilitation $(n=5)$. Design: Single participant series of baseline intervention experiments. The target behaviour was the donning of a prosthetic limb. Method: We introduce the GUIDE $^{\odot}$ - General User Interface for Disorders of Execution. The system provides instructional support for the performance of behavioural sequences, with checks on step achievement and a flexible action protocol controlled by patient feedback. Results: Preliminary data will be reported. We demonstrate disorganised behaviour without the system and normalisation of the sequence when using the GUIDE $^{\odot}$. Discussion: We foresee the use of the GUIDE $^{\odot}$ in neurorehabilitation to aid acquisition and performance of activities of daily living. Food and drink preparation, personal health regimes, exercise regimes may all be suitable applications. We will discuss the system as a platform for errorless learning.

\section{The Use of Biofeedback in Neuropsychological Rehabilitation}

I. Keller

Neurological Clinic Bad Aibling, Germany he implementation of multimedia biofeedback systems offer new
possibilities for the rehabilitation of brain-injured patients. Examples of 3 different approaches for the use of biofeedback in neuropsychological rehabilitation will be presented. The first approach deals with the application 
of neurofeedback for the remediation of attention deficits. It has been shown that the use of neurofeedback as an operant conditioning paradigm can improve cognitive functions in healthy participants (Vernon et al., 2003) as well as brain-injured patients (Hoffman \& Stockdale, 1996; Keller, 2001). Data from a group study and single cases will be presented indicating that a training of EEG beta-activity or a combination of EEG theta/beta training enhances arousal and sustained attention. The second experiment is an interdisciplinary approach in which patients with upper extremity weakness were randomly allocated to either constraint-induced movement therapy (CIMT) or CIMT plus EMG-biofeedback. Patients of both groups received the same quantity of therapy sessions and performed exactly the same movement exercises. Preliminary results show that patients treated with CIMT plus biofeedbacks achieve higher posttraining scores in several motor tasks than patients treated with CIMT only. The third application of biofeedback is designed for patients in the persistent vegetative state. Feedback of the EMG, skin conductance response and heart rate can provide information about the arousal of the patient and help shaping a stimulation program. Data about differential response-pattern to sensory stimulation with and without emotional valence will be presented.

POSTER SESSIONS (in alphabetical order by first author)

\title{
Effects of Neuropsychological Rehabilitation on Functional Performance of Alzheimer Disease Patients
}

\author{
J. Abrisqueta-Gomez, V.L.D. Vieira, F. Canali, C.S.C. Ponce, A.P.C.O Aguiar \\ and O.F.A. Bueno \\ Psychobiology Department, Universidade Federal de São Paulo/Brain Checkup, Consulting \\ and Research in Cognitive Neurosciences, São Paulo, Brazil
}

U $\begin{aligned} & \text { sing Neuropsychological Rehabilitation (NR) in the treatment of } \\ & \text { progressive disorders such as dementia is a relatively recent approach, }\end{aligned}$ and little research has been done in this respect. A point favor of this approach is that its 'holistic' structure takes into account the patient's emotional needs and social context. Objective: Analyze the effects of NR on functional performance of demented patients. Method: 12 patients with probable initial-to-moderate phase Alzheimer disease, average age 72.3 and schooling 11.9 years, all taking anti-cholinesterases, were tested for cognitive, functional and behavioral performance. Using the Basic Activities of Daily Living Scale (ADL; Katz et al., 1970), caregivers rated 17 patient activities; the maximum score is 48 , and lower scores indicate less functional dependence. Patients then underwent NR for cognitive skills, daily activities training and compensatory strategies. Advice was provided for caregivers. Patients were retested after 12 months treatment. Results: Comparative analysis showed that cognitive scores remained stable while behavioral disorders diminished. ADL scores declined (the average fell from 17.3 to 14.2). Most patients benefited, but ADL response varied (5 patients' scores fell more than 4 points, 3 fell 2 points or less, 2 remained unchanged and 2 rose 2 points). Conclusions: Dementia is progressive but NR may be useful for retarding functional dependence, diminishing behavioral alterations and improving family life. 


\title{
Holistic Neuropsychological Group Rehabilitation in Sweden
}

\author{
A. Bartfai, A. Björkdahl, J. Larsson and S.Söderström \\ Rehabiliteringsmedicinska Universitetskliniken, Stockholm, Sweden
}

\begin{abstract}
Thtroduction: Integrated treatments in the form of cognitive and interpersonal therapies within the context of a comprehensive neuropsychological rehabilitation program have been found efficient. Improvement in community reintegration, functioning, increased social participation and productivity is often substantial and gains may be maintained several years after rehabilitation (Cicerone, et al. 2005). However, there is a limited knowledge concerning the range of effects due to differences between the treatment programs. Method: This evaluation includes data on 171 patients with acquired brain injury (ABI) participating in 3 holistic neuropsychological rehabilitation programs in Sweden (Västerås, Göteborg, Stockholm) between 1997 and 2002. Patients reported comparable level of symptoms, but there were differences in age and gender distribution between the groups. Moreover, the programs differed in duration, content and patient diagnosis. The evaluation consisted of a broad neuropsychological assessment and of a number of questionnaires focussing on everyday problems and coping styles before and after the treatment program. Results: indicated limited effects on the self-report variables but more substantial gains on cognitive evaluations. Patients from one program were followed for 5 years after completing the program and at this point of time $50 \%$ were gainfully employed or pursuing academic endeavours. Conclusion: Holistic neuropsychological group rehabilitation seems to have positive effect on cognitive functioning and society reintegration after $\mathrm{ABI}$ in all the programs. There are differences between the programs that are not self-explanatory. These will be further analysed and discussed.
\end{abstract}

\section{Assessment and Neuropsychological Rehabilitation of Executive Function}

\section{E. Bausela Herreras and O. Sánchez-Armáss Cappello \\ Facultad de Psicología, Universidad Autónoma de San Luis Potosí, México}

$\mathrm{T}$ this research, we focus on assessment of executive function in the adult population, from the point of view of cognitive neuropsycholgy. We present 3 paradigms from which a psychological assessment can be developed: qualitative, quantitative and experimental, with an emphasis on the quantitative paradigm. The quantitative paradigm has several advantages, (e.g., instruments can be checked by several observers, and their reliability can be determined with others instruments). The main disadvantage of this approach is the time required to administer, score and clinically analyse test results. Within the context of the quantitative paradigm, we discuss several instruments, such as the Stroop Test, Wisconsin Card Sort Test, Tower of Hanoi Test, Tower of London Test and Tower of Toronto Test, and their relevance to the assessment and rehabilitation of executive functions. In this context, Harmony and Alcaraz (1987) identify the following aspects of behaviour: the ability to begin and finish a series of actions, the ability to sustain serial interferences, the ability to change a conceptual set, and the ability to alternate between several motor activities. It is necessary to have clear operational definitions of these executive skills, in order to facilitate the design of assessment instruments. The design of neuropsychological rehabilitation protocols must be consistent with neuropsychological assessment principles. These protocols may include interventions to ameliorate those executive abilities, such as planning, problem solving and inhibition of inappropriate responses, which differentiate us from other animals. 


\title{
Effects of Sports Concussion on Postinjury Executive Functions in College Athletes
}

J. Becker

Neurocogntive Diagnostic \& Treatment Clinic, LLC, Danielson Health Centre, CT, United States of America

\begin{abstract}
Clinical evidence suggests even mild concussions can result in neurocognitive changes if the athlete is returned to play prior to full healing, there is a greater risk for subsequent concussions and more serious consequences. Functions such as memory, processing speed and mental clarity have been cited. Postconcussion symptoms can attenuate daily life functions for extended periods without full recovery. The frontal lobes and prefrontal cortex are particularly vulnerable to injury. This is due to abundant interconnectivity to widespread brain areas, and their neuroanatomical location. Diffuse and even distant lesions may manifest in frontal lobe symptoms. These factors have implicated executive function difficulties and/or personality change s/p MTBI. This pilot study attempted to assess effects of sports concussion on executive functions. Executive function attenuation can be remarkable in scholar/athletes. Scheduling, organising, planning and working memory are just some of the areas possibly affected. Such outcomes may account for academic difficulty s/p injury and other difficulties in the college environment. Increased understanding of such effects may be helpful in augmenting the college student's recovery. Preliminary Analysis indicated $71 \%$ of concussed students within the past year $(n=7)$ had a Verbal Memory Composite Score (involving attention and working memory) on ImPACT $\leq$ $20 \% ; 86 \%$ were $\geq 10$ pts. symptomatic. $15 \%$ of Athlete Controls $(n=7)$ were $\leq 20 \%$ on VMC and $43 \% \geq 10$ pts symptomatic. Further analysis is being done with additional measures given: (LNS, Trails B, ECB, COWAT-FAS).
\end{abstract}

\section{Neuropsychological Feedback and Satisfaction With Rehabilitation Make a Significant Difference to Family Caregivers of Patients With Stroke}

\section{M.P. Belciug}

Rehabilitation Program, Chedoke Campus, Psychiatry and Behavioural Neurosciences, McMaster University, Canada

\footnotetext{
$\mathrm{T}$ The paper examines the family caregiver's experience with some of the aspects of the rehabilitation process of the patient with stroke. Following their participation in the feedback session on the patients' neuropsychological test results, 45 family caregivers rated their pre- and postfeedback understanding of the patients' neurocognitive strengths and weaknesses. The caregivers' perceptions of the rehabilitation process - including satisfaction with rehabilitation, expectations regarding the patients' recovery and quality of life, and the support available to them in the community - were also examined with a series of 7-point scales. The results indicate that the neuropsychological feedback enhances very significantly the caregivers' understanding of the patients' neurocognitive strengths and weaknesses. Also, the caregivers' postfeedback understanding of the patients' neurocognitive functioning correlated significantly with the caregivers' understanding of how the patients' neurocognitive losses affect the patients' everyday competencies. Likewise, the caregivers were satisfied with the patients' progress in rehabilitation, felt better prepared to assist the patients to overcome their difficulties, and expected the patients' quality of life to be high. Regression analyses revealed that the caregivers' satisfaction with rehabilitation is the
} 
main determinant of their expectation that the patients will enjoy a good quality of life. The caregivers' perceptions of their effectiveness (i.e., they felt better prepared to assist the patients) and their high expectations regarding the patients' quality of life are discussed from the perspective of the motivation of the caregiver to assume his/her caregiving role. The implications of these findings for nurses, therapists and clinicians involved in stroke rehabilitation are discussed.

\title{
Characteristics of Acquired Brain Injury Patients Who are Considered Fit and Unfit to DriveFrom a Neuropsychological Perspective
}

\author{
J. Berry \\ Department of Clinical Psychology, St Joseph's Hospital, Sydney, Australia
}

Background/Objective: Acquired brain injury (ABI) has the potential to affect a person's ability to drive a motor vehicle safely. Neuropsychological assessment can provide a cognitive perspective on fitness to resume driving. The purpose of this study was to investigate demographic, diagnostic and assessment variables in ABI patients considered fit and unfit to resume driving from a neuropsychological perspective. Method: Retrospective analysis of neuropsychological data from a consecutive series of ABI in- and outpatients referred for neuropsychological assessment of driving capacity in a medical rehabilitation service was undertaken. One-hundred-and-thirteen neuropsychological driving assessments were carried out in a 5.5 year period, 14 of which were reassessments. Of the 99 individuals assessed, 57 were recommended to undergo an OT driving assessment because of borderline or unclear results, 27 were considered unfit to resume driving (UNFIT) and 15 were considered fit to resume driving (FIT). The latter 2 groups were compared across a range of variables. Results: There was no difference between FIT and UNFIT in age $(p=.823)$ or time since event/diagnosis $(p=.178)$. Cerebrovascular accident was overrepresented in UNFIT (70\%) compared to FIT (33\%). Neuropsychological tests that best differentiated between the groups were Rey-Osterrieth Complex Figure Test (Copy) $(p=.000004)$, Digit Symbol $(p=.0002)$, Trail Making Test A $(p=.003)$, Trail Making Test B $(p=.004)$, and Bells Test $(p=.005)$. Conclusion: Significant differences exist in diagnosis and neuropsychological test results but not in age or time since event/diagnosis between ABI patients considered fit and unfit to resume driving from a neuropsychological perspective.

\section{Comparison of Three Different Methods to Estimate Premorbid Intelligence in Spanish Population}

\author{
A. Bilbao, ${ }^{1}$ C. Flórez, ${ }^{1}$ E. Lemon, ${ }^{1}$ G.Mejuto ${ }^{1}$ and I. Bombín ${ }^{2}$ \\ ${ }^{1}$ Centro Estatal de Atención al Daño Cerebral. Madrid, Spain \\ ${ }^{2}$ Reintegra Daño Cerebral. Asturias, Spain
}

bjectives: To develop and compare 3 different methods of estimating
premorbid intelligence in the Spanish population. A demographically based method was compared with 2 methods combining demographic variables with actual functioning measures. Participants and Methods: 1370 subjects of the standardisation sample of the WAIS-III in the Spanish population participated in the development of a demographically based model and a combined method that used demographic variables combined with the 
highest score in the WAIS-III. A reading task (TELEI) that required reading of irregularly pronounced words was developed. This task was first standardised in a sample of 300 subjects and then administered along with WAIS-III to an additional sample of 45 subjects, in order to develop a method of estimating premorbid intelligence based on the resistance of reading abilities to brain damage. The 3 methods were then applied to a sample of 100 brain injury patients including TBI, stroke and anoxia. Patients with aphasia were excluded. Results: Demographic methods were the most reliable method of estimating premorbid intelligence in the overall brain injury population. However combined methods were more accurate that demographic methods when patients were above an 8 in the Rancho Los Amigos Scale of Cognitive Functioning. Conclusions: Different methods have been developed to assess premorbid intelligence in the Spanish population. Each method has its own strengths and weaknesses and the application of one on another should depend on the patient's level of cognitive impairment.

\section{Merlin: A Software Offering Variants of the Errorless Learning Method}

C. Bindschaedler, ${ }^{1}$ L. Perseghini ${ }^{2}$ and M. Aeschlimann ${ }^{1}$

1 Service de Neuropsychologie et de Neuroréhabilitation, CHUV, Switzerland

2 Filière informatique logiciel, HEIG-VD, Switzerland

Grorless learning has been shown to be a valuable method to teach speCific information to patients presenting a variety of deficits such as anterograde or retrograde amnesia, anomia and agnosia. Variations of the method have been proposed. The original method requires the patient to copy (or read or repeat) the entire information during several trials. By contrast, errorless learning combined with fading provides the patient with the entire information during the first cycle but with only partial information to be completed during the next cycles, which makes the patient more active. Other variants, still not very much used, involve pre-exposure of one element of the pair (i.e., presenting the face alone for a few seconds before presenting it in association with the name of the person) or preexposure combined with semantic encoding (i.e., presenting the face alone for a few seconds and asking appreciative judgments before presenting it in association with the name). We developed software that allows the therapist to create a database for many patients or learning sessions tailored for one particular patient. We present the design of the software as well as preliminary comparisons of variants of the method, which is practically relevant and can also be informative as to the issue of the underlying memory processes (implicit or explicit?).

\section{Neuropsychologists or Rehabilitation Professionals? Making Neuropsychology Meaningful in a Community Service}

N. Blake, H. Liddiard, L. Jones-Woodward and K. Hilton

Broad Green Centre, Croydon Primary Care Trust, United Kingdom

$\mathrm{T}$ his service development poster describes the process undertaken in order to address waiting lists and redefine models of neuropsychology practice in a community neuropsychology service. The psychologists had struggled to provide a traditional assessment service to the local outpatient Neurology department while also having key therapeutic roles as integral members of the community neurorehabilitation team. Long waiting lists and confusion 
over roles was undermining morale and stifling clinical expertise. Through a 2-year process of service review, the clinicians have sought to meet waiting list demands while also broadening the remit of service provision to embrace current developments in neuropsychological rehabilitation. This paper addresses the conflict between the traditional role of neuropsychology, as a primarily psycho-diagnostic service, and the demands of working as rehabilitation professionals in a multidisciplinary setting. The paper describes how the service has examined its current practice, introduced change and seeks to put accountability to its clients and referrers at the centre of its model of service delivery. It explores the key strengths of clinical neuropsychology in a multiprofessional community service while also outlining the challenges of maintaining specialist assessment and psychodiagnostic skills. It outlines a model of service delivery that seeks to make maximum use of what is unique to the profession while acknowledging the shared roles in rehabilitation practice. The methods used and insights gained will have relevance for the many professional groups working in neurorehabilitation.

\section{What do they Want and What Do They Get? Clients' Evaluations of Their Neuropsychological Assessment}

N. Blake and K. Hilton

Broad Green Centre, Croydon Primary Care Trust, United Kingdom

This poster session describes an evaluation of the neuropsychology a community-based service. Based on a previous qualitative study (Blake, 2004), this evaluation sought to ascertain the needs and concerns of clients prior to their assessment experience and evaluate the outcome from the clients' perspectives. Twenty-five clients were sent questionnaires prior to their assessment asking 5 open-ended questions: Why were they referred? What did they hope to gain from the assessment? What were their greatest concerns in relation to their condition? In what ways would they like to change as a result of their assessment? Were they affected by the waiting time for assessment? The pre-assessment questionnaire, collected at the first interview, was used for collaborative negotiation of the assessment goals. The postassessment data was collected by telephone after the client received their assessment report. Data analysis used the method derived from Interpretative Phenomenological Analysis and a number of core themes were identified. Clients main concerns centred on understanding the reasons for their cognitive problems, being helped to cope practically and emotionally with their problems, addressing issues related to confidence and selfesteem and ways of living with uncertainty. The postassessment data indicates that most clients benefit from clarification of their areas of difficulty and education on strategies that can be easily used in everyday life. Feedback is highly appreciated, with written feedback facilitating recall and reinforcement of strategies. Verbal feedback is perceived as supportive and person-centred. The educational/therapeutic role of assessment is discussed. 


\title{
The Relationship Between Objective and Subjective Memory Assessments in Adults With Brain Injury
}

\author{
P. Blythe, ${ }^{1}$ N. Lincoln ${ }^{1}$ and R. Das Nair ${ }^{2}$ \\ ${ }^{1}$ Institute of Work Health and Organisations, University of Nottingham, United Kingdom \\ ${ }^{2}$ Nottingham University, United Kingdom
}

\begin{abstract}
Durpose: Previous literature, exploring the relation between objective and subjective memory assessments and everyday memory, has achieved disparate findings. The aim of the study was to examine the relationship between the Doors and People (D\&P), an objective memory assessment, the Everyday Memory Questionnaire (EMQ), a subjective measure of everyday memory and the Extended Rivermead Behavioural Memory Test (RBMT-E) an objective measure of everyday memory. Method: A sample of 42 braindamaged adults was recruited as part of a randomised controlled trial of memory rehabilitation. Data was analysed using nonparametric statistics. Results: Highly significant correlations were observed between 6 subtests of the RBMT-E and the D\&P. Limited evidence was found for a significant relationship between the measures of everyday memory, the RBMT-E and the EMQ. Significant relationships were established for the story (immediate) subtest of the RBMT-E and the memory for activities and retrieval factors of the EMQ. Mood was also found to significantly positively correlate with the EMQ. Conclusion: It was concluded that, until further research is completed, both objective assessments and subjective measures of everyday memory should be used in clinical practice in order to gain an accurate representation of an individual's memory functioning.
\end{abstract}

\section{Learning to Use New Technology in an Apartment for Transitional Living}

I.-L. Boman, A. Granqvist, C. Lindberg, A. Bartfai and H. Hemmingsson

Rehabilitation Medicine University Clinic, Danderyd Hospital, Sweden and

Department of Neurobiology, Care Sciences and Society, Karolinska Institute, Sweden

This study investigated the possibilities for patients with cognitive impairments after acquired brain injury (ABI) to learn how to use a set of electronic aids to daily living, (EADL) in an apartment for transitional living. Participants: 16 patients with moderate or severe memory impairment after ABI in a chronic rehabilitation phase were included. Study setting: An apartment for transitional living was equipped with a set of advanced EADL with features covering safety and security issues, keeping track of people, keeping track of schedule and keeping track of medication. Intervention: Each patient stayed for 5 days in the apartment to learn how to use EADL. An individually learning program was designed, based on the patients own experience, habits and wishes identified in an interview, but at the same time considering the participants disabilities, needs and requirements. Data collection: A structured observation protocol was developed in order to evaluate the patient's ability to learn and use EADL. Observations were conducted all days along during the stay. All events and actions were logged and saved in form of lists by the integrated overview system. Results: All patients benefited from the adapted environment and they learned how to use EADL in only 5 days. Implications: If patients can learn how to use EADL during the rehabilitation period they will be less dependent on spouses and caregivers in everyday activities after discharge from the hospital. It can also contribute to develop appropriate EADL that are useful for persons with cognitive impairments. 


\title{
Measurement of Post-TBI Recovery to 12 Months Using Rivermead Post Concussion Symptoms Questionnaire (RPCSQ) Factor Scores: Effects of Clinical and Demographic Variables
}

\author{
P. Cannan, C.E. Skilbeck and M. Slatyer \\ School of Psychology, University of Tasmania, Australia \\ Neurotrauma Register, Royal Hobart Hospital, Tasmania, Australia
}

\begin{abstract}
$O$ bjective: Many TBI sequelae and their recovery are poorly understood. Factor analyses of the RPCSQ yielded 4 factors: emotional, cognitive, physical, and visual. The study's aim was to chart recovery over 12 months post-TBI using these factors, and to examine their relationships with demographic and clinical variables to predict symptom recovery. Method: A population sample of 430 individuals presenting with TBI at the Department of Emergency Medicine, Royal Hobart Hospital participated. Data were collected on the RPCSQ within 1 week of TBI, and at 14 days, 28 days, 3 months, 6 months, and 12 months postinjury. ANOVAs were conducted on RPCSQ factor scores, and Multiple Regression (MR) was used to predict factor outcomes at 6 and 12 months using initial clinical (factor scores, PTA, pain, fatigue) and demographic (age, sex, NART IQ) variables. Results: Participants with longer PTAs reported more symptoms on the factors, as did women. Individuals aged 41-60 years reported the highest symptomatology and those over 60 years the lowest. MR analyses indicated that initial factor scores, age of injury, and pain helped predict physical $\left(R^{2}=.458\right)$ and cognitive $\left(R^{2}=.276\right)$ outcomes, with initial factors score and estimated premorbid IQ assisting prediction of emotional symptoms $\left(R^{2}=.357\right)$ at 6 months postinjury. Discussion: While the results for sex and PTA were expected, the finding of more symptoms in middle-aged participants was surprising (perhaps attributable to the compounding effects of other life stresses). MR analyses help clinicians to predict outcomes. This better understanding should assist in planning and evaluating treatment interventions post-TBI.
\end{abstract}

\section{Effects of a Holistic Rehabilitation Program on Executive Dysfunction in Patients With Acquired Brain Injury}
A. Caracuel-Romero, ${ }^{1}$ G. Cuberos-Urbano, ${ }^{2}$ A. Verdejo-García, ${ }^{2}$ M.A. Coín-Mejías ${ }^{2}$ and M. Pérez-García ${ }^{3}$
${ }^{1}$ FIBAO, Virgen de las Nieves Hospital, Granada, Spain
2 School of Psychology, University of Granada, Spain
${ }^{3}$ School of Psychology and Institute of Neuroscience, University of Granada, Spain

Tntroduction: Executive function deficits are a frequent correlate of
acquired brain injury (ABI). There are several traditional neuropsycholog-
ical instruments to assess executive cognitive functions. However, in the last
years, several self-report scales have been developed to assess the
behavioural and emotional aspects of executive dysfunction and their impact
on activities of daily living. The Frontal Systems Behaviour Scale (FrSBe)
is aimed to assess behavioural deficits associated with damage to frontal-
subcortical systems, whereas the European Brain Injury Questionnaire
(EBIQ) is aimed to assess global consequences of brain injury. Both instru-
ments include a subscale assessing executive dysfunction. Objectives: To
assess the results of a holistic rehabilitation program on executive function
improvement using the FrSBe and the EBIQ as outcome measures.
Participants and Methods: 12 patients with ABI undergoing holistic reha-
bilitation in Granada (Spain). We selected the FrSBe total and executive 
dysfunction scales and the EBIQ total and executive dysfunction scales as outcome variables. Results: We found statistically significant improvements between pre- and posttreatment assessment on the FrSBe and EBIQ total scores and on the FrSBe executive dysfunction scores, but not on the EBIQ executive dysfunction scale. Conclusions: Patients with ABI improved global and executive functioning during rehabilitation. The FrSBe dysexecutive scale is more sensitive than the EBIQ's one to assess executive functioning changes during rehabilitation.

\title{
Increased Use of Memory Strategies Following Attendance at a Single-Session 'Memory Group' Intervention
}

\author{
A.J. Champion, E.L. Hale, A.J. Fletcher and A.S. Thomson \\ Health Psychology Department, Gloucestershire Royal Hospital, United Kingdom
}

\begin{abstract}
bjectives: Preliminary data presented in 2005 indicated that a singlesession memory rehabilitation group intervention increased attendees' use of memory strategies at 1-month follow-up. As such, these sessions have been run regularly with almost 100 patients attending to date. Data from these attendees is presented to evaluate further the utility of this intervention. Methods: The intervention consists of a single session lasting approximately 2 hours; the first section includes sufficient theoretical information to provide a rationale for the second section, which covers 15 external and internal memory strategies. Attendees were consecutive referrals representing a range of aetiologies. Pre- and postintervention self-report measures of anxiety and depression, memory lapses and use of memory strategies were administered. Results: Over $80 \%$ of attendees reported increased use of memory strategies following the intervention. Paired $t$ tests suggest a statistically significant increase in the total number of memory strategies used per week at 1 -month follow-up $(p<.01)$, which represents both a statistically significant increase in the use of external memory strategies $(p<.01)$ and internal memory strategies $(p<.01)$. Conclusions: The results suggest that a single-session group intervention providing information about memory difficulties and describing/demonstrating the use of compensatory strategies is related to increased use of memory strategies at one-month follow-up. This suggests that it is a worthwhile use of limited rehabilitation resources in an acute general hospital setting.
\end{abstract}

\section{The Efficacy of Memory Training in Early Alzheimer Disease}

\author{
V.T. Cruz, ${ }^{1}$ J. Pais, ${ }^{1}$ A. Teixeira, ${ }^{1}$ J.M. Roriz, ${ }^{2}$ N. Antunes ${ }^{1}$ and B. Nunes ${ }^{2}$ \\ ${ }^{1}$ Neurology Department, Hospital de São Sebastião, Santa Maria da Feira, Portugal \\ ${ }^{2}$ Neurology Department, Hospital Pedro Hispano, ULS Matosinhos, Santa Maria da Feira, Portugal
}

Thtroduction: Cognitive training is a nonpharmacological intervention in
Alzheimer's disease (AD) whose purpose is to optimise cognitive func-
tioning, attempting to delay the decline. Objective: Evaluate the efficacy of
a memory training program in patients with early AD medicated with
Donepezil. Methods: Patients with early AD were selected and divided in 2
groups: with/without training. All participants had less than 18 months since
first symptoms and were medicated with Donepezil for less than 6 months.
A global cognitive evaluation was made initially and each 6 months until 18
months of study, using the Lisbon Battery for Assessing Dementia, Blessed
Dementia Scale, Geriatric Depression Scale and the Rivermead Behavioural 
Memory Test. For the stimulation group was defined a period of weekly intervention with 6 weeks duration, repeat after a period of 6 months without intervention. Results: 19 patients ( $7 \mathrm{men} / 12$ women) completed the first 6 months of the study. Seven patients were included in-group without training and 12 in-group with training. Average age was 70 years $(S D=7.9)$; average education was $3.7(S D=1.8)$, with no significant differences between the 2 groups. There were no significant differences in cognitive tests results in the first evaluation and after 6 months intervention, within groups and between groups. A positive difference in the trained skills was found. Conclusions: The initial 6 months of this study showed no significant differences in the neuropsychological results or in the performance of daily living activities. There was, however, a tendency to improve in the specific trained skills.

\section{Sociodemographic Profile of a Spanish Sample of Main Caregivers of Patients With Acquired Brain Injury}

G. Cuberos-Urbano, ${ }^{1}$ A. Caracuel-Romero, ${ }^{2}$ A. Verdejo-García, ${ }^{1}$ M.A. Coín-Mejías ${ }^{1}$ and M. Pérez-García ${ }^{3}$

1 School of Psychology, University of Granada, Spain

2 FIBAO, Virgen de las Nieves Hospital, Granada, Spain

${ }^{3}$ School of Psychology and Institute of Neuroscience, University of Granada, Spain

Tntroduction: Neuropsychology has traditionally emphasised the study of brain functions and the development of assessment tools very focused on patients with brain injury. However, the family system (patient's caregivers) has not received as much attention until the last years, in spite of warnings from important researchers. Most assumptions and traditional reflections about the caregivers stem from the findings of a small group of authors. Objectives: To obtain a sociodemographic profile of the main caregiver of a group of patients with acquired brain injury (ABI). Participants and Methods: The sample was composed of 30 main caregivers of patients with ABI (26 women and 4 males) following neuropsychological holistic rehabilitation in the city of Granada (Spain). Average time as caregivers was 20 months. The information was collected using a novel questionnaire designed specifically for the study. The principal variables were: gender, educational level, age, economic income and kinship. Results and Conclusions: The results indicate that the average profile of the principal caregivers of ABI patients is: 50-year-old woman, with primary studies ( 8 years of education), housewife, couple of the affected one, and with 2 children. The income of the family is approximately $12,000 €$ per year including the economic compensation assigned to the ABI patient. As for domestic tasks, they do not have any type of help, and the role of secondary caregivers is developed by their children. 


\title{
Is There a Role for Mental Practice in Speech Rehabilitation?
}

C. Davis, ${ }^{1}$ D. Farias, ${ }^{1}$ K. Baynes, ${ }^{2,3}$ V. Chand ${ }^{4}$ and O. Orgun ${ }^{4}$

${ }^{7}$ Department of Physical Medicine and Rehabilitation, University of California at Davis, United States of America

${ }^{2}$ Center for Neuroscience, University of California at Davis, United States of America

${ }^{3}$ VA Northern California Health System, University of California at Davis, United States of America

${ }^{4}$ Department of Linguistics, University of California at Davis, United States of America

\begin{abstract}
praxia of speech (AOS) is a complex difficult-to-treat disorder. It is gener$\mathbf{A}_{\text {ally thought of as a breakdown in the motor programming/planning pro- }}$ cess of speech production. Yet, traditional approaches to treatment of AOS have incorporated motor execution into therapeutic activities despite the consensus that the breakdown probably occurs prior to speech execution. The consequence of including motor execution is the unavoidable production of errors, which may inadvertently strengthen unwanted neural-motor pathways. One method of reducing production errors is to discourage speech and require mental practice during rehabilitation. Mental practice, mental imagery and implicit practice are used to describe techniques in which the individual thinks about a task without actual execution and have received much more attention in the fields of sports psychology and physical and occupational therapy. Recent fMRI research has shown mentally simulated tasks to activate sensorimotor cortical areas comparable to overt actions (Ehrsson, et al., 2003; Johnson et al., 2002; Nair, et al. 2003). New treatments that use errorless or implicit therapy in aphasia show that improvements can be made behaviourally that are associated with increases in appropriate activation in the left frontal cortex on imaging (Davis, Harrington \& Baynes, 2006). AOS appears to be particularly well suited to this intervention and has been successfully applied in a case study (Davis, Farias, \& Baynes, 2006). There are significant differences in the models on which motor and speech rehabilitation are based that will have implications for the way these methods are applied to different populations.
\end{abstract}

\section{Analysis of the Activity: Nourishment and Occupational Therapy}

G. Del Moral Orro, M. Fernández Doblado, I. Vico Díaz and J. Bernabeu Verdú

Centro Sociosanitario Nuestra Sra del Carmen, Unidad Daño Cerebral, Valencia Red Menni de Servicios de Daño Cerebral, Valencia, Spain

$\mathrm{N}$ ourishment is one of the most basic activities of daily life due to its vital importance. The detailed analysis of nourishment activity shows how people functionality level depends on the interaction of many factors. Brain injury may result in (any kind of) deficits, which limit the execution of daily activities. For instance, it may be a handicap in cutlery manipulation due to abnormal muscle tone and poor motor control, or perceptual problems so that the individual can not identify the food or the plate, or he may suffer from apraxia. Moreover, a dysfunction in emotional status may make him lose appetite. Occupational therapists focus on the analysis of all those physical, cognitive, social and emotional components and the context where the activity takes place. The exhaustive assessment of each component is performed with standardised test as: COTNAB, FIM-FAM, LOTCA, as well as the information obtained by clinical observations and the interview with the patient. Also, his social and physical environment provides information to occupational therapists to detect the patient deficits and skills and to establish the goals of the treatment. 


\title{
Prediction of Frontal Impairments of Patients With Traumatic Brain Injury in an Early Rehabilitation Setting
}

E. De Guise, ${ }^{1}$ J. Leblanc, ${ }^{1}$ M. Feyz, ${ }^{1}$ and J. Lamoureux ${ }^{2}$

${ }^{\prime}$ Traumatic Brain Injury Program, McGill University Health Centre-Montreal General Hospital, Canada

${ }^{2}$ Social and Preventive Medicine Department, University of Montreal, Canada

\begin{abstract}
$\Lambda$ ims: Most of the predictive studies found in the literature have considA ered long-term cognitive outcome of patients with traumatic brain injury (TBI). Only a few have looked at short-term outcome. The objective of this study was to isolate factors that would predict short-term frontal impairments in TBI patients. Methods: 348 patients admitted to a tertiary trauma centre in an early rehabilitation program participated in the study. Predictive variables were the patient's age and education, GCS (Glasgow Coma Scale), duration of posttraumatic amnesia (PTA) as well as results of cerebral imaging and presence or not of neurosurgical intervention. The frontal impairments were predicted at 3 weeks posttrauma and were assessed with the behaviour components of the Neurobehavioral Rating Scale-Revised (NRSR) (inattention/reduced alertness, agitation, liability of mood, irritability and disinhibition), the mental manipulation test and the Trail Making Test. Results: The results showed that the GCS was the best predictor of behavioural impairments (18.2\%) and the length of PTA was the best predictor of executive function impairments as assessed with the mental manipulation test $(18.5 \%)$ and on the trail B (23\%). In conclusion, these results will help health professionals and administrators to plan more expediently specific services to these patients presenting a more severe TBI and with longer PTA. Knowing the probability of behaviour deficits will also allow clinicians to advise patients and significant others of ways to better deal with the sequelae in this domain and to improve the quality of life.
\end{abstract}

\section{The Influence of Occupational Gaps on Life Satisfaction After Acquired Brain Injury}

\section{G. Eriksson \\ Division of Occupational Therapy, Department of Neurobiology, Caring Sciences and Society, Karolinska Institutet, Sweden}

\begin{abstract}
$A$ $\mathrm{im}$ : To explore the influence of occupational gaps on the life satisfaction for a group of people one or more years after acquiring brain injury. Design: Cross-sectional study by use of a postal survey. Method: A total of 164 persons who had suffered a traumatic brain injury or subarachnoid haemorrhage 1 to 4 years earlier answered a postal survey. It encompassed questions on occupational gaps, life satisfaction and depression as assessed with the Occupational Gaps Questionnaire (OGQ), LiSat-11 checklist and Hospital Anxiety and Depression Scale respectively. Rasch-analyses were used to transform the raw scores from OGQ and LiSat-11 into interval measures, before exploring, through regression analyses, which factors influence the perception of life satisfaction. Results: The extent of the perceived occupational gaps had the greatest impact together with whether or not they suffered depression, on their perception of life satisfaction. These factors explained $31 \%$ and $8 \%$ of the total variance respectively. Whether a person was working or studying after the brain injury explained an additional $2.5 \%$. Implications: The ability to participate in wanted everyday occupations had a strong impact on the perception of life satisfaction one to 4 years after an acquired brain injury. The result indicates the importance of a
\end{abstract}


client-centred rehabilitation process in order to reduce perceived occupational gaps. The OGQ was sensitive enough to capture occupational gaps in this sample when used as a postal questionnaire. The clinical relevance of OGQ seems obvious, but its applicability needs to be tested further.

\title{
A Comparative Study Between the Patient Competency Rating Scale Neurorehabilitation and Subtest Sensitive to Executive Deficits
}

E. Gil Orejudo, M.G. Balet, A. Guzmán Gómez, A. Reyero del Río, A. Nagore Casas and J. Tirapu Ustarroz

Centro Lescer de Madrid, Spain

\begin{abstract}
$\Delta \mathrm{im}$ : One of the main problems in neuropsycological rehabilitation of Acquired Brain Injury (ABI) patients is the patient's lack of awareness of cognitive deficits and emotional and behavioural alterations. Different studies have revealed relationships between alteration of executive processes and adequate awareness of deficit, which entails a lack of progress in rehabilitation and difficulty achieving a partially independent and productive life. Our aim is to study possible relationships existing between a questionnaire of conscience: the Patient Competency Rating Scale Neurorehabilitation (PCRS-NR.) and selected neuropsychological tests sensitive to executive deficits, and to analyse how executive dysfunction is related to the degree of awareness of deficit. We hope to compare whether scores obtained in this questionnaire show significant statistical differences in relation to hemispheric location of injury. Method: A sample of 40 patients, aged between 16 and 75 years, in acute phase. The instruments administered were: PCRSNR (patient, family and therapist), WCST and WAIS-III Matrices. The scores of the 3 forms of PCRS-NR are compared to determine if patients with more opposing scores obtain worse scores in the mentioned neuropsychological tests and if specific anatomical areas more frequently seen to affect awareness of deficit can be identified (data obtained from the neuroimagery tests in the medical reports). Results: This study is currently in data analysis, therefore at this time we cannot forward and conclusions.
\end{abstract}

\section{Recovery of Disorders of Self-Awareness in Patients With Traumatic Brain Injury}

B. González, ${ }^{1}$ J.L. Blázquez, ${ }^{1}$ N. Paúl, ${ }^{1,2}$ M. Rios, ${ }^{1,3}$ D. y De Noreña ${ }^{1}$ and M. Pascual ${ }^{1}$

${ }^{1}$ Hospital Beata Maria Ana, Unidad de Daño Cerebral, Madrid, Spain

${ }^{2}$ Departamento de Psiquiatría y Psicología Médica. Facultad de Medicina, Universidad Complutense de Madrid, Spain

${ }^{3}$ Departamento de Psicología Básica, Facultad de Psicología. UNED, Madrid, Spain

D isorders of self-awareness following an acquired brain injury are one of the most common neuropsychological impairments observed in traumatic brain injury (TBI) patients. Impairment of self-awareness has been considered one of the factors predicting cognitive recovery. Furthermore, it represents a major factor that could limit the patient's autonomy capacity in their daily-living activities as well as in their rehabilitation process. Moreover, reduced self-awareness of the influence of deficits over life's patients is a big obstacle for the reincorporation into their previous social and work environment. For this reason, suitable assessment of this deficit and its appropriate management in the rehabilitation process could be the key to successful rehabilitation. Based on all this, we have assessed the influence of neuropsychological rehabilitation on self-awareness in individuals with TBI, in 2 experimental groups: on one hand, individuals receiving 
neuropsychological rehabilitation, and on the other, subjects not receiving any treatment (e.g., lack of medical resources, or who began rehabilitation process several years after damage). We compared the evolution and recovery on self-awareness in both groups. The sample included 30 individuals with TBI and their family members. We used the Spanish version of Patient Competency Rating Scale, 'Índice de Competencia del Paciente'. Our data indicate that the group, which received neuropsychological rehabilitation showed higher self-awareness than the group who did not receive such treatment. Although there are many variables associated with lack of awareness in acquired brain injury patients, neuropsychological rehabilitation is an important factor for their recovery.

\section{Normative Data for Diagnosing Visual Neglect With a New Screening-Test: Cats Test}

T. Haid

Klinischer Neuropsychologe GNP, Klinischer Neuropsychologe GNPÖ, Klinischer \& Gesundheitspsychologe Allgemein beeideter u. gerichtlich zertifizierter Sachverständiger

Datients with visual neglect demonstrate typical limitations in visual exploration: ipsilesional start of exploration, contralesional omission, and often spatially disorganised exploratory patterns. The authors developed and standardised a simple clinical test, the 'Cats Test': 240 silhouettes (dogs, houses, cats, aeroplanes, clocks, etc.) are scattered on a DIN A4 sheet of paper (width $29.7 \mathrm{~cm}$, height $21 \mathrm{~cm}$ ). The target stimuli, 24 cats, are located only on either the left and right $40 \%$ of the page, 12 per side; diverting silhouettes render the search more difficult. For standardisation, the authors tested 90 self-reportedly healthy volunteers $(27-85$ years of age, $50 \%>67$ years). The number and positions of omissions, position of the first 4 cats crossed out, and exploration time were recorded. The subjects omitted a maximum of 5 targets ( $48 \%$ omitted none, $98 \%$ omitted at most 3); a maximum of 3 targets were overlooked on either side of the page, the difference between left- and right-sided omissions never exceeded 3 cats; $87 \%$ of the subjects crossed out at least one of the first 4 targets on the left half of the page. Correlation of age with the number of omissions was low $(r=.221 ; p$ $=.036$ ). Time of exploration onset did not correlate significantly with age and number of omissions. The Cats Test is a short standardised test for the diagnosis of visual neglect, useful especially for the elderly patients. Test forms, information, and statistical data are obtainable free of charge by e-mail (thomas.haid@tilak.at).

\section{Satisfaction With Life and Quality of Relationship in Partners of Chronically Brain-Injured Individuals}

E. Hämmerling and C. Wendel

Asklepios Hospital Schaufling, Department of Neurological and Neuropsychological Rehabilitation

Rackground: Head injuries do not only change an individual's life but ${ }_{3}$ most often result in a 'head-injured family'. Especially partners experience high levels of burden being caught in between their roles as caring relative and loving partner. Based on a conceptual model of caregiver's stress (Pearlin et al., 1990), this study examined the satisfaction with life as well as the perceived quality of relationship in partners of chronically braininjured individuals and their psychological, as well as care-related, burden. 
Method: 67 partners were examined using the 'Fragebogen zur Lebenszufriedenheit' (FLZ) and the 'Paarklimaskalen' (PKS) in order to evaluate the satisfaction with life and the climate of the relationship. Scales of the 'Symptom Checklist 90-Revised' (SCL-90-R), the 'Screen for Caregiver Burden' (SCB) and semantic differential ratings of the braininjured person's attributes were employed to collect data on psychological and care-related burden. Results: In contrast to most similar studies, this explorative pilot study presents results, which include relatives who do not mainly care in a narrow sense but support their partners psychologically and by structuring everyday life. Regression analyses showed that quality of relationship, as well as satisfaction with life, was predicted only by subjective stressors. Discussion: These results highlight the importance of subjective stressors for perceived burden in relatives of individuals with chronic brain injuries. They emphasise the necessity of ensuring interventions, especially developed for relatives of chronically sick people. A socially legitimate and thus financial foundation for such interventions has yet to be laid in Germany.

\section{The Family's Positive Experience: What Makes the Difference After a Member Sustains a Traumatic Brain Injury}

H. Harrington

Epworth Healthcare/Bouverie, La Trobe University, Australia

Tntroduction: There has been a growth of attention over the past decades investigating the family's functioning after a family member suffers a traumatic brain injury (TBI). Researchers have identified family members may experience feelings of stress, burden, anxiety and depression. Yet, not all family members are negatively impacted by this event. In fact, recent studies have demonstrated two thirds of families were not depressed. What factors contributed to these family's positive outcomes is not yet identified. This question is explored in the following research project. Aims: The paper aims to highlight what assisted these families to adapt to this life-changing event. This information will help guide the field in how best to provide services to assist family members achieve positive outcomes. Method: 12 family members, from differing families, were assessed as not suffering from clinical levels of depression or anxiety. These family members then participated in a focus group interview. Following the principles of grounded theory thematic analysis of the interviews was completed. Results: The study identified family members used a mixture of methods to maintain their family's functioning. Many built upon skills developed when dealing with past family events of loss and trauma. Each described the importance of relationships and making connections with positive people. Central to their process of adapting was the family members' ability to maintain hope, and held the belief their relative had been given a second chance to live. The family members demonstrated a resilience to deal with what life had unexpectedly confronted them with. 


\title{
Self-Instructional Memory Remediation for Paediatric Brain Injury
}

J. Ho, ${ }^{1}$ S. Lah, ${ }^{1}$ A. Epps, ${ }^{2}$ L.Parry ${ }^{2}$ and M. Poole ${ }^{2}$

${ }^{1}$ Department of Psychology, University of Sydney, Sydney, Australia

${ }^{2}$ Brain Injury Rehabilitation Program, Sydney Children's Hospital, Sydney, Australia

\begin{abstract}
The aim of this project is to evaluate a memory remediation program (diary and self-instructional training-SIT) in children with acquired brain injury. SIT is a cognitive top down model that has been used to improve attention in impulsive children as well as adults with attentional deficits. More recently, it has been used in conjunction with diary training and has notably improved everyday memory in adults with brain injury. Developmental literature suggests that children are limited in terms of their ability to develop strategies, and the knowledge to use strategies spontaneously. Preliminary data collected at the Sydney Children's Hospital (Randwick) suggests that the 6-week memory program has been effective in increasing diary use over the short term as well as improving children's perceptions of their everyday memory abilities. Further analyses will be conducted to examine the efficacy of the program over a longer ( 2 month) term.
\end{abstract}

\section{'What Sense Has My Life After Brain Injury?' Neuro-Psychotherapeutic Implications for the Process of Coping With Effects of Brain Injury}

\author{
H. Hofer, H.J. Znoj and R. Müri
}

Universitätsspital Bern-Inselspital Bern, Abteilung für neuropsychologische Rehabilitation Bern, Switzerland

G motional adjustment disorders are a significant and often limiting factor

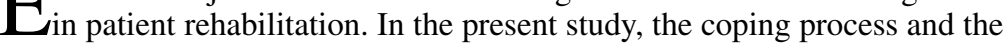
efficiency of psychotherapy in the management of grief after brain injury will be investigated. The preliminary data indicates that in the early phase, the most important coping strategies were 'religion', 'rumination' and 'cognitive coping strategies'. During the course of therapy, a significant difference in central tendencies emerged between the first and second monitoring stage $(p<.05)$. After receiving psychotherapy, patients exhibited 'rumination' less often and 'searching for social support' and 'cognitive coping strategies' more often than at the beginning of the therapy. The central elements of coping strategies were acceptance of the impairment, adjustment to a changed lifestyle and redefinition of reality. Key therapeutic interventions should be problem actuation and clarification as well as developing ways of overcoming them (mastery-oriented) and activating all available resources. Providing information relevant to the condition - such as increased fatigue following a brain injury - also form an essential part of the treatment. From our results, the conclusion can be drawn that psychotherapy with braininjured patients is only helpful if both the perspective of neurorehabilitation and that of psychotherapy are taken into account equally. This will allow patients to improve self-awareness and hence to realistically evaluate their deficits and residual capacity to reestablish more satisfactory interpersonal relationships and to find a new sense in life. 


\title{
Measurement of Mood Recovery in the First Year Post-TBI Using Hospital Anxiety and Depression (HADS) Factor Scores, and the Effects of Clinical and Demographic Variables
}

\author{
K. Holm, C.E. Skilbeck, M. Slatyer and T. Bell \\ School of Psychology, University of Tasmania, Australia Neurotrauma Register, Royal Hobart Hospital, \\ Tasmania, Australia
}

\begin{abstract}
$O^{\prime}$ bjective: Mood disturbance post-TBI and its recovery are poorly understood. Using the 3-factor structure of the RPCSQ (anxiety, depression, psychomotor), the study's aim was to chart mood change in the first year post-TBI. A second aim was to examine mood relationships with demographic and clinical variables to predict symptom recovery. Method: A population sample of 430 individuals presenting with TBI at a Department of Emergency Medicine participated. Data were collected on the HADS within 1 week of TBI, and at 14 days, 28 days, 3 months, 6 months, and 12 months postinjury. ANOVAs were conducted on HADS factor scores, and Multiple Regression (MR) was used to predict mood factor outcomes at 6 and 12 months using initial clinical (factor scores, PTA) and demographic (age, sex) variables. Results: Participants with longer PTAs reported more symptoms on the factors, as did women and individuals aged 41-60 years. MR analyses indicated that each initial factor score was the best predictor of itself at 6 and 12 months post-TBI, with age, PTA, and sex also contributing significantly (range of $R \mathrm{~s}=0.5$ to 0.72 ). Prediction of anxiety factor score was the most accurate. Discussion: While the results for sex and PTA were expected, the finding of more symptoms in middle-aged participants was surprising (perhaps reflecting the combining effects of other life stresses). MR analyses should help clinicians to predict mood outcome for individual patients, and should assist in planning and evaluating treatment interventions post-TBI.
\end{abstract}

\section{In Search of an Ecologically Valid Measure of the Dysexecutive Syndrome: Can Virtual Reality Help in Rehabilitation?}

\author{
A. Jansari, ${ }^{1}$ R. Agnew, ${ }^{1}$ S.A. Duncombe, ${ }^{1}$ L.Murphy ${ }^{2}$ and T. Edginton ${ }^{3}$ \\ ${ }^{1}$ School of Psychology, University of East London, United Kingdom \\ ${ }^{2}$ Rehab UK, London, United Kingdom \\ ${ }^{3}$ Westminster University, London, United Kingdom
}

The accurate assessment of individuals with dysexecutive syndrome
(DS) is vital for effective rehabilitation. Although neuropsychological
tests of executive function exist, they are not necessarily ecologically valid
or predictive of real-world adjustment (Shallice \& Burgess, 1991). The
Jansari, Agnew, Akesson \& Murphy (JAAM) paradigm is an office-based
role-playing task constructed to tap the major deficits seen in DS. As well
as a real-world version, the task was also created to be run on a laptop
computer as a Virtual Reality (VR) environment. Four sets of studies using
JAAM have shown that it is: (a) able to significantly discriminate DS
patients from matched controls (NCs), and that the VR findings replicate
the real-world findings; (b) able to pick up gains following rehabilitation;
(c) can replicate to a different set of patients in another country (Australia);
and (d) can be used to explore age-related decline in executive functioning.
Additionally, fine-grained analysis of individual patients' performance on
specific cognitive constructs can reveal impairments, which are often been
missed on formal clinical testing. The findings are discussed with 
reference to ecological validity and clinical utility of experimental paradigms and the possibility of using JAAM to explore theories of frontal functioning in patients with focal surgical lesions.

\title{
Subjective Contributions to Poststroke Depression
}

\author{
L. Jenkins ${ }^{1}$, D. Andrewes ${ }^{1}$, T. Hale ${ }^{2}$, F. Khan ${ }^{2}$ and N. Coetzee ${ }^{1}$ \\ ${ }^{\prime}$ Department of Psychology, The University of Melbourne, Victoria, Australia \\ ${ }^{2}$ Department of Rehabilitation, The Royal Melbourne Hospital, The Royal Park Campus, Victoria, Australia
}

\begin{abstract}
bjective: In this study, a new questionnaire the Subjective Depression Questionnaire (SDQ) was developed to investigate the roles of various perceived contributions to the development of depression. The SDQ assessed the areas of communication difficulties, cognitive dysfunction, physical handicap, emotional dysfunction, concerns about the future, concerns about finances, social functioning, detrimental changes in life roles, and relationships. This study aimed to identify the factors contributing to depression according to the patients' self-report, and validate the SDQ. Subjects: 27 stroke patients and their partners, and a comparison group of 28 amputees and their partners were sampled from a rehabilitation centre. Measures: Depression was assessed according to the Hospital Anxiety and Depression Scale, the General Health Questionnaire-28, and the Emotional and Social Dysfunction Questionnaire. Results: Stroke patients scored higher than amputee patients on all depression measures. The scales of the SDQ were used to predict a Combined Depression score in a stepwise regression for each group. For the stroke group, the Cognitive Difficulties scale was the first to enter the model, $\left(\mathrm{R}^{2}=.53\right)$. The addition of the Emotional Dysfunction scale increased the $\mathrm{R}^{2}$ to .68 . For the amputee group, the Relationships scale was the only scale to enter the model $\left(\mathrm{R}^{2}=.41\right)$. Conclusions: This study identifies the factors of cognition and emotionalism as subjectively contributing to depression in stroke patients. This knowledge is important for the development of therapeutic interventions for stroke patients, which should target the morbid reaction to cognitive deterioration and emotionalism. Implications for rehabilitation are discussed.
\end{abstract}

\section{Holistic Paediatric Rehabilitation Program for Children With Acquired Brain Injury}

\author{
M. Kerminen, O. Honkinen, T. Korhonen, N. Mellenius and H. Miller \\ The Mannerheim League for Child Welfare, Foundation for the Rehabilitation of Children \\ and Young People, Rehabilitation and Development Centre HUVITUS, Yläne, Finland
}

bjective: Holistic Paediatric Rehabilitation Program for Children with
Acquired Brain Injury (HOPE) is a holistic rehabilitation program for
brain-injured children and their families. The goals of the program are to
increase the child's and his/her family's functioning and quality of life along
with increasing collaboration with local authorities. Rehabilitation perspec-
tive is client-centred and emphasises family's resources, strengths and inter-
action. HOPE is the only rehabilitation programme in Finland in which the
whole family participates and a follow-up and regular collaboration with
school are included. This study evaluates the impact of the HOPE-pro-
gramme on neurocognitive and social development of the child and the
coping of the family. The preliminary results of the study were presented in
EACD 2006 in Barcelona. The study group consists of 15 families partici-
pating in the HOPE Program during the years 2005-2007. Ages of the chil- 
dren vary between 4 and 16 years. Methods: In choosing the methods the ecological validity of assessment tools was emphasised. The background information was gathered using basic neuropsychological tests. In addition, Five-to-Fifteen scale, and BRIEF, HIBS, and MPOC questionnaires were filled out by the parents. Classroom performance of the child was evaluated by the child's teacher. Results and Conclusions: Gathered data indicates improvement in executive functions and attention regulation skills of the child during the program. In addition, social skills of the child and emotional wellbeing of the family were improved. Additional data will be gathered.

\title{
Congruency Between Formal Assessment and Self-Report of Memory Function After TBI
}

\author{
J.M. Leathem and R.A. McGill \\ School of Psychology, Massey University, Wellington Campus, New Zealand
}

\begin{abstract}
$A$ ims: To examine the congruency between self-report and formal evaluation of memory competency after traumatic brain injury (TBI). Methods: The scores of 82 people with TBI on the Auditory Verbal Learning Test AVLT (delayed trial) were compared with memory items from the Patient Competency Rating Scale (PCRS; keeping appointments on time, remembering the names of people seen often, daily schedule, important things and what was for dinner last night). Results: Impairment on formal memory assessment (AVLT Trial $7>1 S D$ below average for age) was found for $56.1 \%$ of the sample and on everyday memory items for 62.2 . However, correlations between AVLT and PCRS scores were low and congruency found for only $52.4 \%$ of the sample (AVLT and PCRS both low, 35.4\%; or both average, $17.1 \%)$. For the remainder $(47.6 \%)$, scores on the measures were incongruent, with almost equal numbers low on the AVLT but average on PCRS $(20.7 \%)$ or average on the AVLT but low on PCRS $(26.8 \%)$. Post hoc analysis revealed relationships between congruency, executive function and employment. Discussion: The dissociation between informal and informal assessment of memory is reported again. Further those with intact memory functioning in one or other domain (formal or self-report) were less likely to have higher executive function difficulties and more likely to return to work than those with (and without) difficulty in both domains. Implications of these findings are discussed.
\end{abstract}

\section{Looking Ahead: A Resource to Assist in Return to School After Acquired Brain Injury (ABI)}

W. Levick, M. Frith and R. Smith

John Hunter Children's Hospital, Newcastle, Australia

Veurorehabilitation of children and young people after an acquired brain 1 injury (ABI) usually involves a multidisciplinary team and begins in hospital. The majority of children who sustain a significant ABI return to school. The cooperation of health and education services then becomes a crucial consideration in producing successful rehabilitation outcomes. Health professionals and education staff often have different perspectives. The process of mutual education regarding respective contributions can be problematical. In 2005/2006 Kaleidoscope's paediatric brain injury rehabilitation team took up an opportunity to develop a DVD-based educational resource for teachers and schools. The aim was to create a user-friendly package for schools and 
teachers that would also facilitate our working with them in the rehabilitation of students with an ABI. This presentation will outline the process of planning and production of the DVD and describe its content and potential applications. The finished product includes a DVD and CD. Content includes a 'feature' with professional actors describing a child's journey from hospital back to the classroom and playground. It contains interviews with team members (e.g., neuropsychologist, speech pathologist) explaining their roles, interviews with parents describing the challenges experienced on their child's return to school, and a collection of guides for teachers including curriculum-referenced material that they can print out for ready reference. The guides detail possible difficulties the child may experience (e.g., problems with writing or recalling instructions), possible reasons for such difficulties and strategies the teacher can use to support the child. Initial feedback has been very encouraging.

\title{
International Classification of Functioning, Disability and Health (ICF) in Neurorehabilitation Daily Practice: An Interdisciplinary Project
}

\author{
J. López Sánchez, C. López Pascua, E. Terradillos Azpiroz, A. Guzmán Gómez \\ and L. Barrio Ruiz \\ Centro Lescer, Madrid, Spain
}

\begin{abstract}
$A^{\text {s. }}$ im: An in depth study of the use of the ICF as a method of complete and interdisciplinary evaluation of the acquired brain injury (ABI) patient. The theoretical model guiding evaluation and rehabilitation of this community should be the biopsicosocial approach (based on the ICF), which is concerned with how different health dimensions, together with environmental factors determine the person's independence. A precise evaluation should gather information from all the different disciplines working with ABI patients, that is, occupational therapy, physiotherapy, speech therapy, neuropsychology, medicine, and so on. Each discipline provides complementary information, which creates the individual profile and treatment program. Method: Each ICF item is accompanied by a code specifying the magnitude of the problem. These codes must be related to methods that guarantee objective evaluations. To insure this we established correspondences between standardised test scores each discipline usually uses with the generic ICF scale. In items lacking evaluation instruments, quantification will be established which may be used in a standard manner for different evaluators. Other ICF sections that fail to take indispensable functions in an evaluation into account will be analysed and subsections added to cover those lacking. Results: This is an ongoing investigation in which different ICF applications will be analysed as a tool for statistics, clinical investigation, education and social politics.
\end{abstract}

\section{Improving Social Outcomes Following Brain Injury Through Development of Confident Communication Skills}

J. Mackey, ${ }^{1}$ S. Sloan, ${ }^{2}$ N. Starritt ${ }^{2}$ and J. Douglas ${ }^{3}$

${ }^{1}$ Applied Communication Skills, Melbourne, Australia

2 Osborn Sloan \& Associates, Melbourne, Australia

${ }^{3}$ La Trobe University, Melbourne, Australia

Social isolation is one of the main long-term issues consistently reported $N_{\text {in outcome studies. The inability to maintain established friendships }}$ and form new social relationships may be traced to underlying cognitive-behavioural impairments and the associated loss of pragmatic 
language function. As a consequence, individuals struggle to re-integrate into social roles and engagement in meaningful occupation reduces. In improving long-term community integration outcomes, social communication skills need to be thoroughly addressed - although these skills are often perceived by therapists as being difficult to teach. The authors will describe how social communication skills can be systematically taught in both individualised and group programs. Therapy activities in core skills of listening, talking, greetings and partings, manners and extending social networks are described. Flexible learning pathways build confidence in social communication, therapy activities are graded from individual activities, practice with a communication partner and then generalised to everyday community activities. The importance of systematically graded practice and the generalisation of skills into specific community roles are illustrated in both individual and group therapy settings. A group intervention involved an 8-week course which focused on the special needs of the ABI population, while being designed using language, format and content that reflected a mainstream training environment. A repeated measures design was used to evaluate course outcomes. Assessment involved a semistructured interview and administration of quantitative measures. Group and individual results will be presented to illustrate the effectiveness of this model of social communication training.

\section{A Simply Self-Sustaining Service in South Africa}

\section{A. Madden}

Community Day Hospitals, Cape Town, South Africa

This presentation describes the evolution of a functional and self-sustaining community-based rural neurorehabilitation service in the Hessequa region of South Africa. The primary focus of this service is on the empowerment of the community in managing and maintaining adults with acquired neurological and neuropsychological impairments in a rural environment. The underlying philosophy is based on the belief that communities and individuals have the inherent capacity to provide the structural and behavioural systems which accommodate impaired persons whereby reducing the need to access primary, secondary and tertiary medical services once the client has been diagnosed as medically stable. The aim of this service is to reduce the dependence on both the medical and traditional therapeutic/hospital models by affording the carers and clients the tools, education and opportunities to assume personal responsibility for achieving relative independence. The service is four-pronged and incorporates: (1) a postacute care short-term individualised hospital-to-home program; (2) a home-based community nursing program; (3) a daily workshop skills-development program; and (4) a palliative service. All patients are managed by family members and carers trained in basic nursing skills who are familiar with the community, the area, and the culture. Expert professional medical and para-medical personnel serve as consultants and trainers at the commencement of the programs and at intervals when required. 


\section{The Mayo Classification System for Traumatic Brain Injury (TBI) Severity}

J.F. Malec, ${ }^{1}$ A.W. Brown, ${ }^{2}$ C.L. Leibson, ${ }^{3}$ J. Testa Flaada, ${ }^{1}$ J.N. Mandrekar, ${ }^{4}$

N.N. Diehl ${ }^{4}$ and P.K. Perkins ${ }^{3}$

${ }^{1}$ Department of Psychiatry and Psychology, Mayo Clinic College of Medicine, Rochester, Minnesota, United States of America

${ }^{2}$ Department of Physical Medicine and Rehabilitation, Mayo Clinic College of Medicine, Rochester, Minnesota, United States of America

${ }^{3}$ Department of Epidemiology, Mayo Clinic College of Medicine, Rochester, Minnesota, United States of America

${ }^{4}$ Department of Biostatistics, Mayo Clinic College of Medicine, Rochester, Minnesota, United States of America

Durpose: To develop a single traumatic brain injury (TBI) severity classification system based on commonly used TBI severity measures and indicators that (1) maximally uses available positive evidence to classify TBI severity in 3 categories: (a) moderate-severe (Definite) TBI, (b) mild (probable) TBI, (c) symptomatic (possible) TBI; (2) reflects current clinical knowledge and relevance; and (3) classifies a larger number of cases than single indicators with reasonable accuracy. Participants: The study sample of a defined population consisted of 1501 unique Olmsted County, Minnesota (US) residents with at least one confirmed TBI event from 1985 to 1999 . Within the sample, 1678 TBI events were confirmed. Main Findings: Single indicators were not available in a large percentage of these events, that is, Glasgow Coma Scale (GCS) was absent in 1242 (74.0\%); loss of consciousness absent in 1178 (70.2\%); posttraumatic amnesia (PTA) absent in 974 (58.1\%); head CT not done in 827 (49.3\%). The Mayo Classification System for TBI Severity was developed to classify cases based on available indicators that include death due to TBI, trauma-related neuroimaging abnormalities, GCS, PTA, loss of consciousness and specified postconcussive symptoms. Using the Mayo system, all cases were classified. For the moderate-severe (definite) TBI classification, estimated sensitivity was $89 \%$ and estimated specificity was $98 \%$. Conclusions: By maximally using relevant available positive evidence, the Mayo system classifies a larger number of cases than single indicator systems with reasonable accuracy. This system may be of use in retrospective research and for determination of TBI severity for planning postacute clinical care.

\section{Development and Validation of a Measure of Processes in Physiotherapy Services}

F. Medina i Mirapeix, ${ }^{1}$ J. Montilla Herrador, ${ }^{1}$ S.L. de Oliveira Sousa, ${ }^{2}$

M.C. Lillo Navarro ,2 P. Escolar Reina ${ }^{1}$ and F. Gómez Arnaldos ${ }^{2}$

${ }^{1}$ Department of Physiotherapy, Murcia University, Spain

${ }^{2}$ Department of Pathology and Surgery, Miguel Hernández University, Spain

Durpose: To describe the development of a self-report questionnaire, the Parents Reports on Physiotherapy Services-Processes (PROPHYS-P), designed to find out how parents of children with developmental problems perceive the physiotherapy services they and their child receive. Methods: Various study designs were developed for identification of relevant domains, pretesting and pilot testing. Identification of domains was from a previous qualitative study using focus groups with parents. Pretesting was developed by means of an interview and a cognitive test with 10 parents whose children were seen at 3 local early intervention centres. Pilot testing was conducted by a survey on 450 parents who attended physiotherapy services in 18 early 
intervention centres. Physiotherapist in each centre gave the questionnaire to each family receiving physiotherapy. Questionnaires were confidentially filled out by the main caregiver and returned by mail to university. Various statistical procedures were used to assess the reliability and the validity of the items and the subscales. Results: Contents of the questionnaire are relevant and comprehensible to parents in pretesting and pilot testing. Its validity was show by factor analysis and positive relations of each item and of its 5 subscales with satisfaction. From the content of items in each factor we defined 5 interpretative labels (information and support to parents, teaching of exercises and follow-up, coordination of care, treat, and context for exchange of information). The instrument and subscales are internally consistent. Conclusions: The PROPHYS-P is a generic instrument that can be used for clinical, quality assurance and research purposes.

\section{Parents' Perception of the Physiotherapy Services in Early Intervention Centres}

F. Medina i Mirapeix, ${ }^{1}$ J. Montilla Herrador, ${ }^{1}$ S.L. de Oliveira Sousa, ${ }^{2}$ M.C. Lillo Navarro, ${ }^{2}$ P. Escolar Reina ${ }^{1}$ and F. Gómez Arnaldos ${ }^{2}$

${ }^{1}$ Department of Physiotherapy, Murcia University, Spain

${ }^{2}$ Department of Pathology and Surgery, Miguel Hernández University, Spain

Purpose: To determine the perception of parents of children treated in early intervention centres, of the quality of physiotherapy received, and to identify areas for improvement. Methods: Subjects of study were parents of the children who attended early intervention centres in Spain. The instrument was the questionnaire Parents Reports on Physiotherapy ServicesProcesses (PROPHYS-P), that has 5 subscales with questions on the perception of facts or circumstances that the parents consider outstanding. Results: In 10 of the 21 items of the questionnaire, at least a fifth of the parents $(20 \%)$ indicated that circumstances happened with an inadequate frequency. The main aspects perceived as problems of quality by the parents are relative to the subscale 'teaching of exercises and follow up' (44.1\%) and 'coordination of care' (22.2\%). The 'treat' (1.9\%) and 'information and support to parents' (9.9\%) subscales are the ones that presented smaller proportion of problems. Conclusions: According to the perception of the parents, physiotherapy services in early intervention centres presents important problems of quality in areas of teaching of exercises and follow-up and coordination of care.

\section{Satisfaction With Physiotherapy Services and Parents' Exhaustion of Children With Development Problems}

F. Medina i Mirapeix, ${ }^{1}$ J. Montilla Herrador, ${ }^{1}$ S.L. de Oliveira Sousa, ${ }^{2}$

M.C. Lillo Navarro, ${ }^{2}$ P. Escolar Reina ${ }^{1}$ and F. Gómez Arnaldos F. ${ }^{2}$

${ }^{7}$ Department of Physiotherapy, Murcia University, Spain

${ }^{2}$ Department of Pathology and Surgery, Miguel Hernández University, Spain

Purpose: To describe and compare parents' satisfaction with physiother-
apy services they and their child receive in early intervention centres
between groups with high and nonhigh degree of exhaustion. Methods: A
cross sectional study was used. 238 caregivers of children with develop-
mental problems responded to a survey to determine their exhaustion and
satisfaction level. Satisfaction was measured by means the 1-dimensional
scale $(0-10)$. Exhaustion was measured by means of 3 adapted items from 
subscale patient/client burnout of Copenhagen Burnout Inventory. Items are related to physical and emotional exhaustion and responses are along a 5-point Likert scale, ranging from 100 (a very high degree) to 0 (to a very low degree). Items were internally consistent and a total score was calculated. Participants were classified as high degree when their total score was higher than percentile 66 and nonhigh level when did not. Student's test was used to compare the mean satisfaction of the group with high degree of exhaustion with the group nonhigh degree. Means differences and their confidence intervals were also calculated. Results: Mean of satisfaction was $8.6(S D=1.5)$ and mean of exhaustion score was $43.4(S D=20.8)$. Thirty-three per cent of the caregivers were classified with a high degree of exhaustion. Exhaustion and satisfaction showed significant relationships $(p<.01)$. Caregivers with high degree of exhaustion had lower satisfaction with physiotherapy services than caregivers with nonhigh degree. Conclusions: Caregivers of children with developmental problems experience good satisfaction and exhaustion. Satisfaction should be addressed by therapists to minimise parents' exhaustion of children with development problems.

\section{Categorical Memory Strategies in Children With Congenital and Acquired Brain Damage: Implications for Cognitive Rehabilitation}

C.B. Mello, M. Muszkat, M.C. Miranda and O.F.A. Bueno

Universidade Federal de São Paulo-SP, Brazil

Tn school-age period, children become able to use categorical memory strategies. Changes from nonstrategic to strategic behaviour in memory performance occur between ages 9 to 10 . If brain injury occurs around this age, it may be expected to impair performance in these developing skills. In this study, we investigated categorical memory strategies in 4, 10- to 14year-old brain-damaged children, 2 of congenital onset and 2 acquired in school-age period, by means of 3 free-recall tests of 30 figures classifiable into different categories and colours: the first immediately after stimuli presentation, the second 20 minutes after a free sorting test (where the preference for conceptual or perceptual stimuli organisation was examined), and a third following a verbal exposure to the category labels. Three groups of 7-, 10- and 14-year-old normally developing children $(N=30)$ were also investigated. Conventional neuropsychological tests were also employed. A $z$ test allowed comparing performance of patients and that obtained by control groups, in which a preference for conceptual organisation $\left(\chi^{2}=172 ; p<.001\right)$ and an increase of the number of items recalled $\left(F_{2,106}=310,8 ; p<.001\right)$ according to age was observed. Both congenital onset patients showed a performance similar to controls in stimuli organisation as well as in recall tasks. In the remaining patients, impairment was identified in delayed ( $z$-scores: $-3,03 ;-1,96)$ and cued recall $(z$ scores: $-3,4 ;-3,43)$, and also a preference for perceptual organisation. Such data suggest that the development of categorical memory strategies is affected by acquired brain damage. These findings may have implications for cognitive rehabilitation planning and for discussions about neuroplasticity. 


\title{
A Systematic Review of the Ecological Validity of Neuropsychological Tests of Executive Dysfunction
}

D. Millar, ${ }^{1}$ J.J. Evans, ${ }^{2}$ and M. Nimmo ${ }^{2}$

${ }^{1}$ Northumberland Tyne \& Wear NHS Trust, Regional Neurological Rehabilitation Centre, Newcastle upon Tyne, United Kingdom

${ }^{2}$ University of Glasgow, Section of Psychological Medicine Academic Centre, Gartnavel Royal Hospital, Glasgow, United Kingdom

\begin{abstract}
f cognitive abilities executive functioning has proven the most difficult to characterise due to its complexities and hierarchical position over other more basic cognitive processes. This conceptual difficulty is reflected in clinical practice where assessment of executive functioning remains problematic. In particular, the extent to which performance on neuropsychological tests reflects the types of problems that confront people in everyday life. Theoretical understanding of executive features, neuroanatomical correlates, and cognitive models of executive functioning is, however, becoming clearer and this provides the opportunity to establish a framework with which to operationalise research. The current review systematically evaluated the agreement between neuropsychological tests of executive functioning and 'everyday' difficulties with a focus on the extent to which research has used theoretical foundations as the basis for testable hypotheses. Thirty-one papers were selected whose major focus was the relationship between neuropsychological tests of executive dysfunction and a measure of 'real life' functioning. The findings suggest that neuropsychological tests of executive abilities do have some predictive ability in terms of general everyday functioning. Research, however, made little use of theory as the basis for explicit hypotheses and this may at least partially have resulted in the limited and inconclusive findings in relation to the agreement between specific 'everyday' characteristics of executive dysfunction and particular neuropsychological tests. Future research should pay greater attention to the development of theoretically based hypotheses and standardised assessment tools that place demands on components of executive functioning using tasks that clearly relate to performance on everyday activities.
\end{abstract}

\section{Sustained Attention in Children With ADHD and Learning Disabilities}

M.C. Miranda, M. Muszkat, A.C. Rosseto, C.C. Rodrigues, T. Barbosa, E.G. Sinnes, L.F. Coelho, R. Andrade, D. Pinheiro and O.A.F. Bueno

Universidade Federal de São Paulo - Escola Paulista de Medicina, São Paulo, Brasil

bjectives: To investigate the performance of children with Attention
Deficit/Hyperactivity Disorder (ADHD) and Learning Disabilities (LD)
in the Conners' Continuous Performance Test from normative data obtained
from a sample of Brazilian children. Participants and Methods: The
Brazilian normative sample was taken from the previous study. The clinical
groups were composed of 23 children with ADHD and 32 children with LD,
who were diagnosed by a multidisciplinary team. The performance on the
measures of the Conners' CPT was evaluated using the generalised linear
models for the variance analysis, and using the least squares regression
method corrected by sex and age. Results: The results showed significant
differences between the ADHD and LD group on the percentage of omis-
sions, HIT RT, Hit RT SE and variability, the ADHD group presented a
poorer performance. In comparison, the children with ADHD from the
Brazilian standardisation sample presented a poorer performance in almost 
all measures of the test, except on the percentage of commissions, detectability and response style. The group with LD scored a higher percentage of omissions and commissions, HIT RT SE, variability, perseverations, and lower detectability than the children from the Brazilian group of standardisation. Conclusion: In spite of the sample of children with ADHD being small, it was observed that the measures of Conner's CPT-II distinguish clinical and nonclinical groups, similarly to the data obtained from the American population. But mainly it was observed in this study the deficit patterns in children with ADHD and LD on a sustained attention test.

\title{
Improvement in Everyday Memory: Multifactorial Memory Training Program
}

P. Montejo, M. Montenegro, J.M. Ruiz and M. Llanero

Centro de Prevención del Deterioro Cognitivo. Madrid Salud. Ayuntamiento de Madrid, Madrid, Spain

\begin{abstract}
Tntroduction: The decrease in cognitive performance and specially in some 1 types of memory with aging has been confirmed by many studies. Several interventions with profitable results have been carried out to cope with this deficit. A multifactorial memory training program (UMAM method), has been carried out for elderly in the City Council of Madrid. Objectives: To assess the effects of multifactorial training in memory and other variables. Methods - Subjects: 3862, mean age 69.52 \pm 4,89. Assessment: Pre and post Mini Examen Cognoscitivo (MEC), Geriatric Depression Scale (GDS), Rivermead Behavioural Memory Test (RBMT), Associated Pairs, Memory Failures Everyday (MFE). Training: Multifactorial program of 11 sessions in a group of 14 people lasting 90 minutes each. Results: Global improvement: 35\%; 69\% subjects improve. Normal Memory Pre: 24.7\%; Normal Memory Post: 54.7\%. anova repeated measures: RBMT Pre: $7.91(S D=$ 2.19); RBMT Post: $9.33(S D=2.15) p<.001$; Associated Pairs, Pre: 13.98 $(S D=4.90)$; Associated Pairs, Post: $16.19(S D=5,05) p<.001$; GDS Pre: $10.10(S D=6.02)$ GDS Post: $8.12(S D=5.62) p<.001$. MFE Pre: 22.12 $(S D=9.08)$; MFE Fin: $17.00(S D=8.51) p<.001$. We analyse everyday memory data. Conclusions: the results of this study show that the memory program yields improvement in objective memory and in memory complaints. Besides, it achieves benefits in mood.
\end{abstract}

\section{Evaluating Categorical Effects: Items and Norms Collected from Healthy Spanish}

\author{
F. J. Moreno-Martínez, ${ }^{1}$ K.R. Laws ${ }^{2}$ and P.R. Montoro ${ }^{1}$ \\ ${ }^{1}$ Departamento de Psicología Básica I, UNED, Madrid, Spain \\ 2 School of Psychology, University of Hertfordshire, United Kingdom
}

$\mathrm{T}$ The organisation of semantic memory is a debated issue for cognitive neuropsychologist. One of the main paradigms guiding semantic memory research is the study of categorical effects: the selective impairment of knowledge in one domain (e.g., living things) with respect to another intact - or comparatively, less affected - knowledge in other domains (e.g., nonliving things) or vice versa. In this work, we will present normative cognitive and psycholinguistic data for 140 color photographs belonging to 7 living thing (LT), and 7 nonliving thing (NLT) categories. The items will be standardised in a group of 100 healthy Spanish controls on the following variables: age of acquisition, familiarity, manipulability, naming, typicality, and visual complexity. As in other normative studies, participants will be evaluated (with a Likert type scale) on the above-mentioned variables. We will calculate internal global consistency of each factor (reliability) with 
Cronbach coefficient $\alpha$, as well as validity of the study by comparing our results with other similar studies calculating correlations among the factors. We believe our norms will provide an interesting methodological tool for Spanish researchers. These items will be useful in studies on semantic category-specific impairment in Spanish population, particularly in Alzheimer's disease, as well as in other brain pathologies. Currently there are not many normative studies available in Spanish, which have focused on semantic memory assessment.

\title{
Risk of Psychiatric Disorder in Children With Congenital versus Acquired Brain Dysfunction
}

L. Mounce, ${ }^{1}$ S. Wall, ${ }^{1}$ I. Frampton, ${ }^{1,2}$ J. Tonks, ${ }^{1}$ and P. Yates ${ }^{1,3}$

${ }^{1}$ Centre for Clinical Neuropsychological Research, School of Psychology, University of Exeter, United Kingdom

2 Paediatric Liaison Psychology Team, Cornwall Partnership NHS Trust, United Kingdom

${ }^{3}$ Mardon Neurorehabilitation Centre, Exeter, Devon, United Kingdom

\begin{abstract}
Qjectives: Previous epidemiological studies into the prevalence of psychiatric disorders have demonstrated a greatly elevated risk following brain injury in childhood. 1 in 10 children and adolescents in the United Kingdom (UK) were found to have at least one psychiatric disorder in a recent governmental survey (Meltzer et al., 2000). However, children with brain-injury were not assessed. Using a refined screening measure, this study investigated the risk of psychiatric disturbance in children with congenital brain dysfunction and those with acquired brain injury (ABI), a previously unexplored comparison. Additionally, the study aimed to determine whether there is an 'early vulnerability' of the immature brain, with greater deficits following injury at a younger age. Method: Strengths and Difficulties Questionnaire (SDQ) responses were collected from parents of children with congenital brain-injury $(n=23)$, ABI $(n=23)$ and normative controls $(n=$ 23 ). This permitted assessment of emotional and conduct problems, hyperactivity/inattention and peer relation difficulties. All groups were matched for age and gender. Results: An elevated prevalence of abnormal behaviour and greater disturbance as rated by the SDQ was found in brain-injured children relative to controls across all SDQ scales. Overall difficulties reached clinical significance in $73.9 \%$ of the congenital group and $61.9 \%$ of children with ABI compared to just $4.3 \%$ of controls. The congenital group had greater risk of psychiatric disorder than children with ABI, particularly for emotionality and conduct problems. Conclusions: Results lend tentative support to the early vulnerability hypothesis. The practical implications of findings for service planning are discussed.
\end{abstract}

\section{Verbal IQ and Performance IQ in Spina Bifida and Hydrocephalus: Nonverbal Learning Disabilities Syndrome}

E. Muñoz Marrón

Asociación Madrileña de Espina Bífida, Madrid, Spain

im: Nonverbal learning disabilities syndrome characterises for the pres-
ence of deficits in motor and haptic skills, alterations in visuospatial pro-
cessing, difficulties in problem solving and concept formation, reaching
better performance in the tasks in which the verbal capacities are involved.
In the present paper we try to determine if this syndrome is present in spina
bifida with the aim to identify strong and weak points in order to plan an 
effective neuropsychological intervention. Methods: The sample is composed of 10 subjects with spina bifida and hydrocephalus, aged between 19 and 41 years ( 2 males and 8 women), users of the Centre for Disabled Physicists of the Association of Madrid of Spina Bifida coordinated with the Community of Madrid. All of them have been assessed with WAIS-III (Wechsler Adult Intelligence Scale-III; Wechsler, 1997). From the scores obtained in the test, differences between the various indices have been calculated (verbal comprehension, perceptual organisation, processing speed and working memory), as well as of the general indices: Verbal IQ and performance IQ. Results: Preliminary results support the hypothesis of the existence of nonverbal disabilities syndrome in persons with spina bifida and hydrocephalus, such that verbal IQ was higher than performance IQ. All cognitive areas showed alterations, with speed processing showing the most marked deficit. Furthermore, a worse performance is observed in picture completion, block design, digit symbol-coding and symbol search, all of them subtest that are part of performance IQ.

\section{ADHD and Comorbid: Contribution of P300 in a Case Report}

M. Muszkat, A.G. Rezende, L.D. Pereira, M.C. Miranda, E.G. Sinnes, S. Palma, R.M.B. Andrade and D. Pinheiro

Universidade Federal de São Paulo - Escola Paulista de Medicina, São Paulo, Brasil

bjective: The objective of this study was to assess the changes in latency and amplitude of the cognitive evoked potential P300 in a case of ADHD and comorbidities, aiming to help define the neurophysiological criteria which enable monitoring of the efficiency and the need for multiple drugs in the treatment of ADHD symptoms. Participants and methods: The subject was a child of 13, diagnosed with ADHD, assessed before and after treatment under the following conditions: (a) without medication; (b) with methylphenidate $(20 \mathrm{mg}$ ) only; and (c) with methylphenidate and clozapine. Auditory ERPs were elicited using an oddball paradigm. Results: The combination of methylphenidate and clozapine resulted only in cognitive lethargy without improvement in the academic performance and hyperactive behaviour. The assessment of the P300 pre-medication showed a potential of $305.2 \mathrm{~ms}$ latency and $24.79 \mathrm{uV}$ amplitude; with the use of methylphenidate drops in latency to $288.2 \mathrm{~ms}$ and in amplitude to $14.47 \mathrm{uV}$ were observed. With clozapine, more severe drops in the latency $(273.20 \mathrm{~ms})$ and amplitude $(21.85 \mathrm{uV})$ were observed. The acquirement of P300 with clozapine + methylphenidate resulted in a rise in the latency above the basal levels without treatment, reaching $316.20 \mathrm{~ms}$ with average amplitude of $14.76 \mathrm{uV}$. Conclusion: The prolongment of the P300 latency under the combination of medicaments corresponded to a behavioural response predominantly inhibitory, which agrees with the clinical response observed. Thus, the use of neurophysiological measures such as P300 may be a useful instrument for the selection of drugs for patients with ADHD and psychiatric comorbidities. 


\title{
Memory Without Memory: Assessing the Integrity of Implicit Memory Using the Implicit Memory Test
}

\author{
R.A. Nannery, E. Greenfield, B.A. Wilson, BA, S. Sopena and R. Rous \\ Medical Research Council Cognition and Brain Sciences Unit, Cambridge, United Kingdom
}

\begin{abstract}
$\mathrm{M}$ emory is a faculty with many facets. As a result a neurological insult can affect memory function in different ways, leaving some forms intact while destroying others. There is evidence to suggest that implicit memory is more robust than explicit memory following such damage. However, the means of assessing this in different neurological groups has been inconsistent and often fails to account for differences in conceptual and perceptual processing. The recently published Implicit Memory Test (IMT) aims to allow researchers and clinicians to assess this more subtle form of memory through the use of 2 well documented perceptual implicit memory tasks: the verbal word stem completion and the visual fragmented pictures tasks. The performance of 100 nonacute patients from 4 diagnostic groups (Alzheimer's disease, cerebral vascular accident, traumatic brain injury, and encephalitis) are considered on these verbal and visual implicit tasks and compared to their performance of tests of explicit memory function as assessed on the Rivermead Behavioural Memory Test-III. The differential performance of these groups on perceptual implicit memory tasks suggests that implicit memory for visual stimuli is preserved to a greater extent than that for verbal stimuli. Moreover, in all groups implicit memory is more robust than explicit memory, but the extent to which this is true varies across the diagnostic groups.
\end{abstract}

\section{Psychological Intervention to Relatives of Patients With Severe Traumatic Brain Injury or Severe Subarachnoid Hemorrhage}

\section{A. Norup and L. Siert \\ Unit for Traumatic Brain Injury, Division of Neurorehabilitation Hvidovre University Hospital, Copenhagen, Denmark}

bjective: To investigate whether an extended psychological intervention to relatives, from time of injury until the patient has finished treatment and is discharged from hospital, has an influence on occurrence of anxiety, depression and quality of life. Method: Participants in the clinical randomised research study are relatives of severely brain-injured patients from the Intensive Neurosurgery Unit at Rigshospitalet, Copenhagen (acute phase), who will be sent for further rehabilitation at the Unit for Traumatic Brain Injury, Hvidovre Hospital, Copenhagen (subacute phase). The participants are randomised into an intervention and control group. The intervention group receives psychological intervention in the acute phase, subacute phase and for a follow-up period of 3 months after discharge from hospital. The control group is offered psychological intervention and support in the subacute phase. Both groups complete a questionnaire constructed by the authors with elements from standardised questionnaires. The first part of the questionnaire consists of selected items from SCL-92 (Symptom Checklist) concerning symptoms of anxiety and depression. The second part of the questionnaire consists of selected items from SF-36 (Short Form) regarding quality of life. The third part, a visual analogue scale, represents subjectively perceived quality of life in the relatives. The relatives complete the questionnaire 5 times in the period from time of injury until one year after the injury. Results: The authors expect that 40 relatives will be included in the study. The authors estimate to have collected and analysed data by 2008 . 


\title{
A Comparison of Activity and Content in Compensation, Restitution and Attention Placebo Memory Rehabilitation Groups for Brain-Injured People
}

M.C O'Brien, R. Nair, N. Lincoln and P. Blythe

Institute of Work, Health and Organisations, University of Nottingham, United Kingdom

\begin{abstract}
$A$ ims: The aim of this study was to observe and compare activity and content in different memory rehabilitation groups for participants with brain damage. Methods: Compensation and restitution memory rehabilitation groups and an attention placebo group involving brain-injured participants, enrolled in a larger randomised controlled trial, were observed. Time sampling was used to detail the activity and content in the groups at 1-minute intervals. Based on the time sampling data, activity and content were subsequently compared in each type of group. Results: There was a significant difference between the amounts of time the group leader and participants spent talking across the 3 groups $(P<.001)$. Participants in the compensation and restitution groups spent significantly more time in memory rehabilitation than participants in the attention placebo group $(P<.001)$. There was also a significant difference between the amount of time spent discussing internal and external memory aids in the compensation and restitution groups $(P<.001)$. Conclusions: Each type of group had a different level of input from the group leader and participants. The proportion of time spent in memory rehabilitation in the compensation and restitution groups was high. In contrast, the frequency of nonmemory rehabilitation discussion was high in the attention placebo group. The combination of rehabilitation and nonrehabilitation content in the 2 treatment groups provide insight into the running of neuropsychological rehabilitation groups. The value of process-oriented research in neuropsychological rehabilitation is highlighted.
\end{abstract}

\section{Perception of Speech Errors: Native Perception Versus Formal Acoustic Analysis}

O. Orgun, ${ }^{1}$ V. Chand, ${ }^{1}$ D. Farias, ${ }^{2}$ C. Davis ${ }^{2}$ and K. Baynes ${ }^{3,4}$

${ }^{1}$ Department of Linguistics, University of California, Davis, California, United States of America

${ }^{2}$ Department of Physical Medicine and Rehabilitation, University of California, Davis, California,

United States of America

${ }^{3}$ Center for Neuroscience, University of California, Davis, California, United States of America

${ }^{4}$ VA Northern California Health Care System, University of California, Davis, California,

United States of America

bjective: In speech rehabilitation, interventions may be formulated based on the types of difficulties that a given patient has, with articulatory deficiencies often identified via native speaker/listener (NSL) perceptions. However, NSL perceptions are influenced by the contrasts and phonetic categories of their native language: these thus influence the categorisation of errors. While not problematic in structural linguistics, where NSL judgments are the method of choice, there may be pitfalls when perceptual judgments guide the choice of intervention. Participants and Methods: We present 2 specific examples from an apraxic patient being treated at the UC Davis Medical Center. To better understand the nature of the speech errors, tokens of the patient's speech were compared with tokens from a NSL, with waveform, length and spectrographic features analysed within Praat acoustic analysis software. Our first example involves word initial affricates. In words such as 'joke', NSLs had labelled the patient's pronunciation as incorrectly devoicing the initial affricate (such that it sounded like 'choke'). The second example concerns word initial [s] plus plosive 
clusters (sk..., sp..., st...). In these clusters, NSL linguists perceived the plosive as aspirated (in English, plosives are normally not aspirated in this position). Results: Through acoustic analysis, it was shown that neither perceived error matched the patient's actual pronunciation. Instead, the patient's problem in each case was timing. Conclusions: Since disordered speech may contain phonetic realisations not found in normal speech, it is important to use instrumental analysis to identify articulatory problems when planning interventions.

\title{
Rehabilitation of Social Communication Disorders: A Discussion Paper on Developments in Brain Injury Rehabilitation
}

\section{Prince}

The Oliver Zangwill Centre, Ely, United Kingdom

\begin{abstract}
Therapy techniques and outcomes of intervention for social communication difficulties in people with learning disability and autistic spectrum disorder have been well documented. Very little, however, has been reported about the techniques and outcomes of intervention for clients with social communication difficulties following an acquired brain injury. We have in recent years, reviewed our approach to social cognition, in particular developing an approach to assessment of this area. This discussion paper introduces some of the speech and language therapy intervention techniques, which are being experimented with at the Oliver Zangwill Centre in the rehabilitation of clients with social communication difficulties as a consequence of an acquired brain injury. Assessment and interventions are discussed in relation to 2 clients who attended the centre for a 6-month holistic neuropsychological rehabilitation program. Both cases emphasise the importance of detailed assessment, and demonstrate that some benefit from education can be achieved, although further research needs to investigate consistency across larger populations, in both an experimental and control groups. The cases discussed also demonstrate the diverse nature of the assessment findings and suggest that certain impairments are perhaps easier to relearn than others. Challenges to rehabilitation, including realising generalisation of achievements into everyday life, and developing compensatory strategies to manage social interactions are discussed in the face of the unpredictable nature of social interaction.
\end{abstract}

\section{Post Concussion Syndrome Longitudinal Effects and Management}

R.J. Rees

Disability Research, School of Medicine, Flinders University, Australia

This paper identifies persistent post concussion syndrome (PCS) in a group of 20 adults (10 males and 10 females) age range 18-52 years mean CA 31.9yrs.. This was a hard core group whose PCS lasted for a mean of 3.4 years. Subjects identified sensory, somatic, affective and cognitive PCS symptoms immediately following their trauma (01) and 2 years later (02). Likewise they completed the Beck Depression Inventory (BDI-II) at 01 and 02 . Counselling and psychotherapy interventions took place between 01 and 02 individually every 2 weeks. Items on the PCS schedules and the BDIII inventory were rank ordered and paired sample $t$ tests were calculated to ascertain significant changes in PCS and BDI-II items and related behaviours. Results demonstrated a significant decline in the presence of 
overall symptoms at the end of 2 years, most noticeably in agitation, irritability, and suicidal orientation. However, subjects throughout generally experienced the feeling that they were being punished which equated with behaviours comparable with a state of learned helplessness. The group are not malingerers, but post trauma became 'different people'. Modification of goals and lifestyle, of relationships and employment options gradually changed their dependent state in the flow phase. Comparability with other conditions such as PTSD and chronic fatigue syndrome (CFS) was demonstrated by individuals who experienced the most persistent and invasive post concussion symptoms.

\title{
Community Rehabilitation for People With Brain Injury: Poster Presentation
}

\author{
R. J. Rees and M. Bellon \\ Disability Research, School of Medicine, Flinders University, Australia
}

Sixty people with severe brain participate each year in a university-based $\boldsymbol{N}$ community rehabilitation and learning program funded by State government and research grants. Participants are assessed and referred by health professionals. The program has a manager and deputy, a clinical director, workshop coordinators and management board. Each person with brain injury has a mentor who helps to: organise and support their domestic and social life, attend weekly workshops and camps, participate in individual counselling where necessary. Five separate 3-hour workshops - communication and language skills, movement and personal awareness, computing skills, social activities and problem solving, fine arts; drama/painting, photography, music, are held each week. Residential camps take place every 2 months. The program creates an 'in-house' community, which establishes friendships and networks as a basis for successful participation within the wider community. Participants identify with and 'own' the program demonstrating that friendships, skills and confidence are best developed in a 'safe' community where interests and motivation are enhanced by sustained participation in activities for which the person with brain injury has responsibility. Responsibilities include preparation for workshops and camps, maintaining contact, developing social awareness, contributing to program newsletter and program planning. All important executive functions are nurtured by careful planning so a person's talents and optimism are enhanced.

\section{Formulating the Couple Relationship Before and After Severe Closed Head Injury and Exploiting Neuropsychology and Specialist Head Injury Counselling Resources in Treatment}

L. Russell and D. Aronson

Bath Neurorehabilitation Services, Royal National Hospital for Rheumatic Diseases NHS Foundation Trust, Bath, United Kingdom

$\mathrm{T}_{\mathrm{c}}^{\mathrm{k}}$ There is a great deal of evidence that brain injury in one partner places a considerable strain on the marital relationship. Researchers have explored this in 2 main ways - by looking at the factors in the brain-injured person that have contributed the greatest reported stress for spouses or by exploring the coping histories and practices that distinguish couples who cope successfully from those who do not. Opportunities to work directly with couples to look at both sets of issues are often limited by time and service availability. This case study describes the work done jointly using 
neuropsychology and counselling resources with a couple 18 months after the husband, who had suffered a very severe brain injury, was discharged from intensive early-stage rehabilitation. The formulation incorporated neurobehavioural, dynamic, systemic and cognitive-behavioural concepts and was developed conjointly between the neuropsychologist, the counsellor and the couple. Exploration of pre-existing patterns of coping with conflict and stress, as well as pre-existing self-esteem issues were explored and tracked forward through the brain injury and beyond. The interacting roles of personality factors, cognitive changes and changed circumstances were delineated in order to develop the formulation. The formulation aimed to enable the couple and the therapists to understand the current difficulties and to provide a context for exploring changes. The format of the sessions and work set between sessions were designed to take account of both the impact of the neurobehavioural consequences of the injury and the individual nature of the couple's relationship.

\title{
The Relationship between Clients' Cognitive Functioning and the Therapeutic Working Alliance in Postacute Brain Injury Rehabilitation
}

\author{
M. Schönberger, ${ }^{1,2}$ F. Humle ${ }^{1}$ and T.W. Teasdale ${ }^{2}$ \\ ${ }^{1}$ Center for Rehabilitation of Brain Injury, University of Copenhagen, Denmark \\ 2 Department of Psychology, University of Copenhagen, Denmark
}

\begin{abstract}
bjective: To examine the impact of brain-injured patients' cognitive abilities on their working alliance with their therapist in postacute rehabilitation. Design: Cognitive tests were administered to brain-injured individuals at the beginning of postacute, holistic brain-injury rehabilitation. Clients as well as their primary therapists rated their mutual working alliance at 4 time points throughout a 14 weeks rehabilitation programme. Subjects: 86 clients as well as their primary therapists. Clients had suffered a traumatic brain injury $(n=27)$, a cerebrovascular accident $(n=49)$ or another neurological insult $(n=10)$. Measures: (1) Neuropsychological tests of attention, memory and higher cognitive functions; and (2) the Working Alliance Inventory, client and therapist form. Results: Overall, the relationships between cognitive tests and working alliance ratings were weak. The tests of attention, memory and higher cortical functions were differentially related to clients' and therapists view on their mutual therapeutic alliance at the different stages of their collaborative work. Discussion and Conclusion: Clients' cognitive profile affects clients' and therapists' view on their working alliance in different ways. The weakness of the correlations between cognitive tests and working alliance ratings may indicate that a good working alliance is achievable also with clients with severe cognitive difficulties.
\end{abstract}

\section{The Relationship between Physical Fitness and the Follow-Up Outcome of Postacute Brain Injury Rehabilitation}

M. Schönberger, ${ }^{1,2}$ N.Hansen ${ }^{1,2}$ and D.T. Pedersen ${ }^{1}$

${ }^{1}$ Centre for Rehabilitation of Brain Injury, University Of Copenhagen, Denmark

2 Department of Psychology, University Of Copenhagen, Denmark

bjective: To investigate the relationship between brain-injured patients'
physical fitness and the follow-up outcome of postacute brain injury rehabilitation. Design: One-group interventional study, measurement of 
physical fitness pre- and posttraining, cross-sectional follow-up. Subjects: were 98 patients who underwent a holistic neuropsychological outpatient rehabilitation program. Patients had suffered a traumatic brain injury $(n=26)$, a cerebrovascular accident $(n=58)$ or another neurological insult $(n$ $=14)$. Intervention: 1.5 hours of intensive training of cardiovascular fitness and muscle strength 1-3 times weekly as part of a 3-month holistic, interdisciplinary rehabilitation programme. Measures: Physical fitness was measured with the Harvard step test, the Åstrand cycling test, and patients' walking/running speed. As measures of follow-up outcome, the type of patients' employment and the amount of working hours were registered. Results: Overall, good physical fitness was related to return to competitive, full-time employment. Fitness test results from programme end tended to predict work integration better than test results from programme start. The Harvard step test was the best predictor, followed by the walking/running test. Discussion: Physical fitness is an important factor for work integration. Fitness training may have the most beneficial effect with regard to work integration for those patients who are not in the physical condition, but have the potential to return to a competitive work environment at full time.

\title{
Proposal of a New Method for Exploring Impairments of Arithmetic Facts Retrieval Processes
}

M. Sobanska

Laboratory of Cognitive Neuropsychology, Faculty of Psychology, Warsaw University, Warsaw, Poland

The study presents a method, which allows calculating the amount of time required to work out the result of the arithmetic problem (relative RT) independently of encoding-production mechanisms. It is assumed that the patterns of relative RTs in different experimental conditions will help identify the impaired processes of arithmetic fact retrieval. The method of the relative RTs was used to explore the relationships between the pattern of relative RTs and lesion localisation. We compared the calculation performance of 2 clinical groups (LHD vs. RHD) in 4 experimental conditions, which differed in format of stimuli presentation (Arabic digits vs. spoken number words) and format of response (Arabic digits vs. spoken number words). Eighty-one multiplication problems (MULT) to solve were presented visually or auditorily to each participant. In order to neutralise time consumed for encoding and production processes the subjects were presented with the transcoding control task (CTRL) compound of 4 analogues blocks. The response latencies in CTRL were then used to calculate the relative RTs in the MULT. Divergent patterns of the relative RTs were observed in each group. Our explanation of this finding is that the impairment affected different processes of multiplication fact retrieval in the 2 groups. The method of calculating the relative RTs turned out to be sensitive to the lesion localisation, however, further research is required to explore its clinical utility.

\section{Remediation of Reading Difficulties Based on Successive and Simultaneous Processing: A Case Study}

\author{
M. Shukla and B.R. Kar
}

Centre for Behavioural and Cognitive Sciences, University of Allahabad, India

$\mathrm{R}$ emediation for reading difficulties could train with specific reading skills or could work on underlying processes such as simultaneous and 
successive processing found deficient in children with reading difficulties. We discuss 2 case reports referring to 2 children identified with reading difficulties who were taken for remediation (a 10-year-old boy studying in grade IV and a 12-year-old boy studying in grade V). Both were found well below average on simultaneous and successive processing on the Cognitive Assessment System (CAS). PASS Reading Enhancement Program (PREP) based on the Planning, Attention, Simultaneous and Successive Processing Theory was employed. PREP aims at developing information processing strategies for successive and simultaneous process, deficits in either of the 2 may lead to poor visual and phonological decoding. Global process training and curriculum related bridging training in PREP facilitates application of internalised strategies used for word decoding and spelling. PREP was conducted for thirty sessions for each child. Both the children showed greater advantage from global to bridging component on simultaneous tasks as compared to successive tasks. More sessions were needed for tasks that required visuo-spatial analysis. Improvement from global to bridging component was slower for tasks loaded with temporal processing and working memory. These children were assessed on phoneme oddity and deletion and CAS before and after remediation. Improvement on phoneme oddity and deletion was observed in terms of better accuracy and reduced RTs. Their performance on CAS showed greater improvement on simultaneous processing as compared to successive processing after remediation.

\title{
Reliability of the European Brain Injury Questionnaire (EBIQ)
}

\author{
S. Sopeña, ${ }^{1}$ T. Teasdale, ${ }^{2}$ R. Nannery, ${ }^{3}$ B.K. Dewar ${ }^{4}$ and B. Wilson ${ }^{1,3}$ \\ ${ }^{7}$ MRC Cognition and Brain Sciences Unit, Cambridge, United Kingdom \\ ${ }^{2}$ Department of Psychology, University of Copenhagen, Denmark \\ ${ }^{3}$ The Oliver Zangwill Centre for Neuropsychological Rehabilitation, Ely, United Kingdom \\ ${ }^{4}$ Institute of Psychiatry, St Thomas' Hospital, London, United Kingdom
}

bjectives: In 1997 Teasdale et al. published the European Brain Injury Questionnaire, a self-report measure concerning the subjective experience of cognitive, emotional and social difficulties designed to be given to people with brain injury and their close relatives. The study discriminated between people with brain injury and controls and between people with stroke or traumatic brain injury. Responses can be analysed in terms of 9 subscales. It is now used in several rehabilitation centres as an outcome measure even though test-retest reliability has not been determined. The primary purpose of this study was to improve our knowledge of the properties of a clinically used questionnaire and to find out whether this test is reliable over time. Methods: The questionnaire was administered twice within a month to people with brain injury, relatives and controls. Results: A significant testretest reliability was evident in all groups. Conclusions: The EBIQ is a clinically reliable measure to determine the subjective well being of people with brain injury and to assess change of subjective concerns over time. Ease of administration and analysis suggests that the questionnaire provides a useful and efficient tool for clinicians to measure different subjective domains in people with brain injury. 


\title{
The Executive Secretariat Task (EST); A Real-Life, Ecologically Valid Measurement of Executive Functioning
}

\author{
J.M. Spikman, A. Hol-Steegstra, H. Rietberg, S. Vos, D. Boelen and K.F. Lamberts
}

Department of Neurology, University Medical Centre Groningen, Postbus, Netherlands

\begin{abstract}
$\mathrm{M}$ easurement of executive functioning (EF) is problematic. Ideal tests should tap the essence of EF, that is, self-initiation, self-structurisation and self-regulation of task performance. In order to be ecologically valid, that is, tapping EF in the same way daily life situations do, tests should also require organisation and prioritisation of multiple tasks over a longer timespan, while dealing with delayed intentions, interruptions and deadlines. Traditional tests are too structured and hence do not measure those executive abilities that make persons effective in the real world (Burgess and Simons, 2005). In order to evaluate effects of treatment for EF problems, we designed a task fulfilling the abovementioned requirements, the EST. In this 3-hour task a job assessment procedure is simulated. The subject is alone in a room with a box in which a series of simple secretarial assignments. These have to be organised and executed, some of them with a deadline. No cues are provided about how to carry out the assignments, but necessary materials and information are all available. At fixed times questions can be asked to the 'chief'. During the assessment, the subject is interrupted with an urgent new assignment. The task yields 3 scores: Initiative, Prospective and Executive, which together form the total score. The task was administered to 70 brain-injured patients with EF deficits and 60 healthy controls. Analyses showed significant differences between both groups, indicating that the task is sensitive to executive problems. Significant correlations with the BADS and DEX showed its concurrent and ecological validity.
\end{abstract}

\section{Recovery of Apraxia After Left Hemisphere Stroke: A Case Study}

V. Stamenova, ${ }^{1}$ E.A. Roy, ${ }^{1,2,3}$ W. Mcllroy ${ }^{1,2}$ and S.E. Black ${ }^{1,4}$

${ }^{1}$ Graduate Department of rehabilitation Science, University of Toronto, Canada

${ }^{2}$ Department of Kinesiology, University of Waterloo, Canada

${ }^{3}$ Department of Psychology, University of Waterloo, Canada

${ }^{4}$ Department of Medicine, University of Toronto, Canada

I imb apraxia is an inability to pantomime and imitate gestures. While Lcommon after stroke, few studies have examined its spontaneous recovery using comprehensive assessments. RM, a 69-year-old right-handed man with left hemisphere infarct, underwent 4 assessments (at 1, 3, 20 and 36 months poststroke), examining tool knowledge and gesture production. His performance scores were transformed to $z$ scores relative to those of agematched controls, and if below -2 ( $2 S D$ s below controls), performance was classified as apractic. The Western Aphasia Battery was used to determine RM's aphasia profile. Initially, RM had severe Wernicke's aphasia (AQ = $42)$, which converted to Anomia ( $A Q=68)$. RM was consistently impaired on all tool-naming tasks, due to his persisting aphasia. Tool identification by function performance was initially impaired $(z=-44)$, but eventually recovered $(z=0)$. Gesture performance was initially impaired on all tasks but also improved. For transitive gestures performance improved on imitation $(z=-9$ to -4$)$, on pantomime to tool $(z=-11$ to -4$)$, to function $(z=-17$ to -2$)$, to picture $(z=-12$ to -3$)$ and on object use $(z=-6$ to 0$)$. Pantomime $(z=-13$ to -6$)$ and imitation $(z=-42$ to -1$)$ of intransitive gestures also improved. While RM improved on all tasks, he remained impaired on all tasks except 
object use and imitation of intransitive gestures. RM's apraxia persisted into the chronic stage, but recovery rate differed between conceptual and production scores, suggesting a dissociation between the 2 systems. Studies are underway to determine generalisability, but rehabilitative techniques are clearly needed to enhance recovery in such patients.

\title{
Provision of Rehabilitation Services Needs to Take Account of the Multiple, Interactive and Cumulative Neurological Events that Produce the Long-Term Cognitive Deficit in Children Treated for Posterior Fossa Tumours
}

\author{
R. Stargatt \\ La Trobe University, Austin Hospital, Murdoch Children's Research Institute, Australia
}

\begin{abstract}
Tntroduction: Considerable cognitive morbidity is reported in children 1 treated for posterior fossa tumour (PFT). Long term effects, commonly with decline over time has significant implications for the provision of rehabilitation services. Aim: The aim of this study is to identify factors that impact cognition in children treated for PFT and identify the nature of change over time. Method: Thirty-five children with PFT were enrolled into a prospective study. Neuropsychological assessment was conducted at diagnosis and at 12 months intervals for 3 years. Results: PFT has an impact on intelligence, attention and information processing skills. Hydrocephalus at diagnostic confers a higher risk of neuropsychological deficits. Cranial Radiation Therapy (CRT) is correlated with a decline in attention, information processing and IQ. Change over time is not necessarily a consistent decline and appears as a complex interaction between insult, recovery, stabilisation and decline. The characteristics of change over time are different for different types of neurological insult. Conclusions: Multiple factors, including initial damage to the cerebellum and surrounding structures, hydrocephalus, CRT and white matter injury contribute to the final cognitive outcome in children treated for PFT. Rehabilitation programs need to take account of the differing requirements of the child with PFT over time.
\end{abstract}

\section{The Efficacy of Periodic Auditory Alerts in Improving Performance on a Virtual Reality Task Of Planning and Prospective Memory}

\author{
S. Sweeney, ${ }^{1}$ D. Kersel, ${ }^{1}$ R.G. Morris ${ }^{2}$ and J. Evans ${ }^{3}$ \\ ${ }^{1}$ The Community Treatment for Brain Injury, Gorbals, United Kingdom \\ ${ }^{2}$ Neuropsychology Unit, Department of Psychology, Institute of Psychiatry, London, United Kingdom \\ ${ }^{3}$ University of Glasgow, United Kingdom
}

(2002), by examining whether presentation of periodic auditory alerts
improves performance on a virtual reality (VR) task. The Removals Task
makes demands on planning and prospective memory skills, and requires
participants to take on the role of a removals person and carry out 4 tasks,
with some tasks needing to be interrupted so that another task could be com-
pleted. Seventeen participants with nonprogressive brain injury, who pre-
sented with executive dysfunction, completed 2 equivalently difficult
versions of the VR task, under 2 conditions: with and without periodic audi-
tory alerts. Seventeen control participants were employed to investigate
equivalence of difficulty of both versions of the Removals Task as well as to
determine whether the task was sensitive to the effects of brain injury.
Analysis of control data revealed that both versions of the Removal Task 
were of equivalent difficulty. The brain-injured group was significantly poorer than controls on some, but not all measures of performance in the Removals Task. Contrary to the findings of Manly et al., there was no significant effect of periodic alerts on the performance of the brain-injured group. We speculate that a difference in the cognitive demands made by the VR based 'Removals Task' compared to the 'Hotel Task' used by Manly et al. may account for our findings. In addition we propose that a more extensive 'goal management training' may have facilitated the function of the auditory alerts.

\title{
Neuropsychological Assessment, Rehabilitation and Transition Planning for an Adolescent With Glutaric Aciduria Type 1
}

E. Talbot, E. Bennett, S. Thomas and A. Starza-Smith

Paediatric Neurosciences, Queen's Medical Centre Campus, Nottingham University Hospitals NHS Trust, United Kingdom

Glutaric Aciduria Type 1 (GA1) is an inherited, autosomal recessive, dehydrogenase. Acute striatal necrosis during infancy, often following recurrent infection, is the principal clinical manifestation in this rare condition. This neurological event results from accumulation of lysine, hydryoxylysine and tryptophan and their immediate breakdown products due to the body's inability to process these amino acids. Severe motor disorder such as dystonia and dyskinesia often pursue due to assumed basal ganglia damage. Although outcome varies greatly due to individual differences in degree of acquired brain injury, severe motor impairment with preserved cognitive functions is commonly observed clinically, and is presented in the limited literature available. This indicates the need for comprehensive planning when considering appropriate rehabilitation pathways and educational provision in individual cases. We introduce the case of a 15 -year-old female with GA1 and associated dystonic, dyskinexic cerebral palsy and seizures. We reveal the innovatively selected neuropsychological assessment battery (including subtests of the new Wechsler Nonverbal Scale of Ability) compiled for this case, used to assess all possible cognitive domains, including memory, executive function and general intellectual abilities. Discussion of the special considerations made in the choice of assessment battery due to severe motor and speech impairment is provided. We present a review of the literature on this condition, the neuropsychological and psychological assessment techniques employed in this case, and finally detailed assessment findings and recommendations for rehabilitation. The importance of this assessment in informing educational provision and transition to adult life is discussed.

\section{Driving Ability Following Brain Injury and the Predictive Value of Neuropsychological Tests}

\author{
T. Teasdale, ${ }^{1}$ A. Meng ${ }^{2}$ and J. Jørgensen ${ }^{3}$ \\ ${ }^{1}$ Department of Psychology, University of Copenhagen, Denmark \\ ${ }^{2}$ Danish Transport Research Institute, Copenhagen, Denmark \\ ${ }^{3}$ Center for Rehabilitation of Brain Injury, University of Copenhagen, Denmark
}

Driving is a highly complex skill involving perceptual, cognitive and psychomotor elements. It also plays a central role in the everyday lives of very many people. Brain injury can compromise all of the skill elements 
involved and postinjury evaluation of driving skills is often essential. Within the context of a postacute rehabilitation program, 45 patients underwent an advisory governmental driving test. Performance was assessed on a number of dimensions including appropriate speed, manoeuvring, road positioning and attention to other vehicles. For only 5 patients was overall performance assessed as unsatisfactory. Performance was weakly related to measures of injury severity. It was unrelated to the presence of aphasia but was markedly poorer in the presence of neglect and right hemisphere injury, with noticeable tendencies to veer towards the left (i.e. middle of the road). Patients also completed a range of neuropsychological tests, both at the beginning and at the end of the rehabilitation program, including Wechsler subtests, Trail-Making, Paced Auditory Serial Addition Test (PASAT) and the Wisconsin Card Sorting Test (WCST). Driving performance was unrelated to the PASAT and WCST but was related to the TrailMaking test and several of the Wechsler subtests. The results are discussed in the context of the predictive validity of neuropsychological tests.

\title{
Effects of a New Computerised Exploration Training on Neural Recovery From Spatial Neglect
}

\author{
M. Thimm, ${ }^{1}$ G.R. Fink ${ }^{2,3}$ and W. Sturm ${ }^{1}$ \\ ${ }^{1}$ Department of Neurology, University Hospital RWTH Aachen, Germany \\ ${ }^{2}$ Department of Neurology, University Hospital Cologne, Germany \\ ${ }^{3}$ Institute of Medicine, Research Centre Jülich, Germany
}

Tn previous studies we have shown that chronic neglect resulting from Iright hemisphere lesions can be ameliorated by alertness training or optokinetic stimulation. The present study investigated the behavioural and neural effects of a new developed computerised training that combines elements of both trainings. In this training patients have to observe lively scenes in the role as a photographer. They have to follow a view finder with their eyes and press a button ('shutter release') to 'take a photo' as fast as possible whenever an object appears in the finder. Six righthemisphere stroke patients presenting with stable hemineglect performed the training, 45 minutes daily for 3 weeks. Neuropsychological tests and fMRI were assessed 2 times before (baseline) and 2 times after (long term effects) the training period. The fMRI paradigm was a stimulus detection task derived from the neglect task of the 'Test Battery for Attention Performance' (TAP). Short term improvements of neglect appeared in the form of less omissions and shorter reaction times of left sided stimuli in the scanner task. This was accompanied by increased neural activity in the left superior frontal gyrus (BA 9) and the left superior parietal lobule (BA 7). By contrast, there was no increased neural activity at the baseline and at the long term fMRI. The results suggest that short term amelioration of neglect can be mediated by enhanced neural activity in homologous brain areas contralateral to the lesioned frontoparietal network for spatial attention. 


\title{
Impact of Establishing Comfort and an Environment for Learning in Recovery From Low Level Conscious States: A Single Case Study
}

\author{
J. Tierney, M. Darcy and J. Mackey, \\ Private Practice, Melbourne, Australia
}

\begin{abstract}
Tntroduction: Diagnosis of a vegetative state for more than 6 months following anoxic brain injury is traditionally considered a permanent condition. There are no standardised long-term management strategies for this group and rehabilitation efforts tend to be nihilistic. This case study is of a 58-year-old man, diagnosed as being in a vegetative state for 13 years following anoxic brain injury at age 43. Method: A management program based around comfort and participation in personal care tasks was introduced 13 years post injury. Initial assessments were undertaken using the Disability Rating Scale (DRS) and Wessex Head Injury Matrix (WHIM). These were readministered at 12 months and 2 years. Intervention: A daily care program was developed around all activities involving manual handling, personal care and positioning. A series of specific movement programs were developed integrating controlled sensory input, with each activity broken down into a specific sequence of steps and sensory input maintained below startle threshold. A training manual and a series of videos were developed to support procedural and errorless learning. Results: DRS and WHIM scores improved at 1-year post intervention and WHIM showed continued improvement at 2 years. Discussion: a long-standing low level conscious state does not preclude possibility of new learning and ability to participate in a rehabilitation program based around everyday activities.
\end{abstract}

\section{Neuropsychological Rehabilitation Outcomes}

\author{
S. Tollman ${ }^{1}$ and A. Watts ${ }^{2}$ \\ ${ }^{1}$ Dept of Psychology; University of KZN, Durban, South Africa \\ 2 Entabeni Hospital, South Ridge Road, Durban, South Africa
}

Teuropsychological rehabilitation aims to reorganise shattered brain link1 ages in a way that functioning will again resonate positively within each individual's particular sociocultural ecosystem. In South Africa there is a dearth of follow-up programs. Our Head Trauma Program in Durban has its roots in that of the Rusk Institute of NYU Medical Centre (Dr Yehuda Ben Yishay). The Durban Program implemented in 1987, is as intensive as South African constraints permit ( $2 \mathrm{hrs} /$ day, $3 \times$ per week with $2 \times 20$ week modules). Philosophy; holistic approach; creation of a 'therapeutic' community remain, followed by in situ training. This present study follows a pilot study (South African Law Journal, May 1993, p. 357 ff). Its scope (in progress) now includes all functions in every domain of living. Objectives: (1) Followup of accessible trainees; (2) Individual script analyses to identify themes. Subjects: Approximately 40 brain-injured trainees that completed the program between 1987 and 1999; aged between 18 and 54 years; and education between Grade 10 and postgraduates. Follow-up: Telephonic interviews with trainees and significant others in progress. Results to date are consistent with reports of considerable success of an holistic neuropsychological program to restore a meaningful life. Important factors emerging for sustaining reintegration include support; implementation of strategic planning skills (frontal systems); self-awareness and environmental synchrony. 


\title{
An Exploratory Study of the Cognitive Correlates of Psychosocial Functioning Following Brain Injury Acquired in Childhood
}

J. Tonks P.J. Yates, W.H. Williams, I. Frampton and A. Slater

School Of Psychology, University Of Exeter, Washington Singer Laboratories, Exeter, United Kingdom

\begin{abstract}
Rackground and Aims: Children with acquired brain injuries (ABI) com${ }$ monly present with a range of difficulties, including emotional, social and behaviour problems, as well as accompanying multiple cognitive impairments. Adult research indicates that impaired executive functioning (EF) negatively impacts upon psychosocial functioning and outcomes following TBI. We explored the role of cognitive correlates of psychosocial functioning in children with head and brain injuries in 2 studies. Methods: In the first study 14 children with head injury aged 8 to 10 years were compared with 24 healthy controls. Between-groups comparisons and correlations were carried out. Parents, teachers and the individual children in both groups were administered the SDQ used as a measures of socioemotional behaviour and psychosocial functioning. A second study was carried out on 14 children with ABI, aged 9 to 16 years, and compared with a control sample of 67 healthy children. Results: In study 1, there were no significant differences between children with head injuries and controls on EF measures. In study 2, children with ABI performed significantly worse than controls on EF measures. Preliminary results from both studies suggest significant differences in psychosocial functioning in both head and brain injury samples when compared to controls. In both studies tests of processing speed correlated with problems in psycho-social functioning. Conclusions: The findings of these studies suggest a relationship between cognitive functioning and psychosocial outcome. The authors will discuss further findings and their implications in this paper.
\end{abstract}

\section{Neuroconstructivistic Intervention for Attention Deficit Hyperactive Disorder: A Comprehensive Approach for its Understanding and Management}

L. Trinidad

Trinidad Institute for Neuropsychological Studies, Bayamon, Puerto Rico

$\mathrm{T}$ The neuroconstructivist approach is a panoptic model to assess, understand and treat brain functions. It is based on the complex and dynamic interaction between internals dispositions or cognitive functions of the human being and his environment. This model of intervention pays attention to neuropsychological aspects and the context of its manifestations. It views the realisation of the interventions as a process of active construction. In the aspect of the Attention Deficit Hyperactivity Disorder (ADHD) executive skill deficits are considered vital in order to describe the disorder. These deficits are related to: self-regulation, metacognition, flexibility, attention, working memory, goal-directed persistence, initiation/inhibition, and planning. The intervention begins with a comprehensive assessment observing a biocultural frame; which embraces the neuropsychological and ecological assessment that helps to understand the manifestation of the disorder. The neuroconstructivist intervention gives a foundation where skills deficits are corrected in a significant context. It integrates the intra and interpersonal components, providing the space for a holistic growth. Interventions are presented according to the child needs and interest considering the interaction with the environment. The child needs, settle the starting module combining 
the participation of parents and teachers. The modules of intervention are: 'Self-awareness and our awareness' (child and child-family-school interactions working on metacognition and self-regulation of affect and behavior), 'Bending' (flexibility, persistence, and initiation/inhibition), 'Focus' (attention and working memory), 'Strategy' (planning). Strategies of intervention and case example will be addressed in this presentation.

\title{
Psychosocial Factors and Cortisol Rhythmicity in Close Relatives of Patients With Severe Acquired Brain Injury
}

\author{
J.M. Turner-Cobb, ${ }^{1}$ J. Palmer, ${ }^{1}$ D. Aronson, ${ }^{2}$ L. Russell ${ }^{2}$ and S. Purnell ${ }^{2}$ \\ ${ }^{1}$ University of Bath, United Kingdom \\ ${ }^{2}$ Bath Neurorehabilitation Services, Royal National Hospital for Rheumatic Diseases, United Kingdom
}

$\boldsymbol{B}$ ackground: The role of the family is vital to patient rehabilitation after $\boldsymbol{B}_{\text {brain injury. Existing research on family coping has been in relation to }}$ psychological rather than physical health. This research specifically addresses both the psychological and health impacts on the close relatives of patients who have suffered a serious brain injury. This is to help inform decisions relating to provision of family support. It examines the links between psychosocial coping and hypothalamic pituitary adrenal (HPA) axis activity in close family members of patients. HPA-axis activity is measured via levels of the stress hormone cortisol. The hypothesis is that cortisol levels will be disrupted in relatives of acquired brain injury patients. These disruptions will be attenuated by psychosocial coping resources, such as coping style and perception of social support. Methodology: Participants provided saliva samples for detection of cortisol levels at 4 intervals throughout the study, taken 4 times a day over 2 days. Questionnaires relating to psychosocial factors were completed at the beginning and the end of the study. Preliminary results: First stage samples indicate an overall raised cortisol level in the relatives of the brain-injured patients. Other findings are a positive correlation for Impact of Events Scale avoidance with evening cortisol. Further results from the evening cortisol samples suggest religious or spiritual beliefs are associated with reduced stress levels and carer strain is associated with increased stress levels.

\section{Neuropsychological Rehabilitation of Mild Brain Injury in Preexisting Bipolar Disease}

B.P. Uzzell

Memorial Neurological Association, Houston, Texas, United States of America

Cientific evidence suggests Neuropsychological Rehabilitation is not successful after mild brain injury in cases with pre-existing bipolar disorder. In this case report a 55-year-old male who had a diagnosis of bipolar disorder for 24 years sustained a mild brain injury. Following injury memory losses, inattentiveness, slow processing speed, word fluency limitations were present. Four months after injury Neuropsychological rehabilitation to improve cognitive functioning was initiated individually along with counseling in an outpatient setting. During treatment medications consisting of lithium carbonate, Inderal and Wellbutin continued. The 6 months of cognitive treatment consisted of attention process retraining and strategies to facilitate short-term recall plus counseling to understand the nature of squelae of mild brain injury and methods for coping with 
changes in activities of daily living. Following treatment a gradual return to work was initiated. With this treatment program mental heath remain stable and the symptoms associated with bipolar disease did not surface. The findings suggest the individuals with mental illnesses can recover without incidences following mild brain injuries.

\title{
Effects of Atomoxetine in a Patient Recovering from Brain Ischemia
}

\author{
P. Valdez, D. Chairez and G. Nava
}

Department of Psychophysiology, School of Psychology, Universidad Autónoma de Nuevo León, Monterrey, México

\begin{abstract}
A tomoxetine is a nonstimulant drug that has been used since 2002 for the management of patients with attention deficit/hyperactivity disorder (ADHD). This study provides evidence that atomoxetine interferes with the capability to follow sequences in a patient recovering from brain ischemia. The patient was a 32-year-old, right-handed man. He had a myocardial infarction that produced brain ischemia, affecting all cortical lobes and basal ganglia. The patient was assessed 1 year after the brain ischemia. He had disorders in attention, memory, and executive functions. After a year working in a treatment program, the patient's attention and executive functions improved, but memory showed no changes. At this time atomoxetine was prescribed, $40 \mathrm{mg}$ daily, at morning. The patient was monitored 6 months before treatment, during the 2-week period under treatment, and 6 months after treatment. Atomoxetine did not affect attention: the patient had the same level of response in a continuous performance test and digits forward; but interfered with the capability to follow sequences: the patient repeated fewer digits backward, generated less words of a semantic category, made more errors in a digit symbol task, reading a paragraph and assembling a jigsaw puzzle. Due to these results, medication was discontinued and the patient recovered completely. The capability to follow sequences is one aspect of executive functions, so it is important to further study possible effects of atomoxetine on these functions.
\end{abstract}

\section{Multimethod Intervention for Children With Cognitive Difficulties After Brain Tumour}

I. van 't Hooft' ${ }^{1}$ and A. Lindahl ${ }^{2}$

'Neuropaediatric Unit, Astrid Lindgren's Children's Hospital, Karolinska University Hospital, Stockholm, Sweden

${ }^{2}$ Child Cancer Research Unit, Karolinska Institute, Stockholm, Sweden

Systematically evaluated methods for rehabilitation of children with neuropsychological and psychosocial difficulties are long awaited by parents as well as neuropsychological rehabilitation professionals. Recently, the Amsterdam Memory and Attention Training for Children (Amat-c) has demonstrated positive outcome in a controlled study involving children with cognitive difficulties after brain tumour. The effects included improvement of behavioural problems related to attention and executive functions. However, the method showed no significant effect on social behaviour. Furthermore, the 17-week program was considered too long. Therefore, a modified program based on the Amat-c is developed and evaluated. The length of the program is reduced to 10 weeks. Furthermore, a parents' support program is added, with the aim of helping the parents to coach the child when generalising the effects of the training. The program is evaluated in a 
pilot study involving 4 children, and adolescents, 9 to 16 years, for whom treatment for brain tumour was completed 1 to 6 years ago. Neuropsychological and psychosocial functions are assessed with established tests and self-report instruments. Baseline tests, posttraining tests, and follow-up tests are performed.

\section{Use of Card and Board Games in Executive Function Remediation in Children}

R. Verdon ${ }^{1}$, W. Levick ${ }^{1,2}$ and K. Drysdale ${ }^{1}$

${ }^{1}$ University of Newcastle, Australia

2 John Hunter Children's Hospital, Newcastle, Australia

This study examined the use of card and board games in the remediation of executive function in children with $\mathrm{ABI}$ or $\mathrm{AD} / \mathrm{HD}$. We reasoned that the use of such games would enable therapy to be delivered in the context of 'normal' interaction with a trainer who could model inhibitory and problemsolving strategies in a way that cannot readily be delivered via computerbased programs. The executive demands of a wide range of children's card and board games were examined and documented so that their use could be tailored to the individual case. In this study parents served as their child's trainer. A training manual was developed and parents were involved in a 2hour training session before commencing work with their children. A selection of tests of attention and executive function commonly used in neuropsychological assessment plus parent and teacher behaviour checklists were used to measure executive function before and after therapy. Three children with $\mathrm{ABI}$ and a group of 15 children with $\mathrm{AD} / \mathrm{HD}$ participated in the study. The AD/HD children were assigned to intervention and no-intervention groups. The program duration was 12 weeks with 6, 30-minute sessions per week. Parents were contacted fortnightly to check on progress and discuss any difficulties. Improvements in performance on a majority of measures occurred with some medium to large effect sizes though due to small numbers results failed to reach statistical significance. The outcomes are discussed in terms of the impact of major therapy variables such as program materials, delivery approaches, duration and timing.

\section{Dysexecutive Problems and Interpersonal Relating Following Frontal Brain Injury: Reformulation and Compensation in Cognitive-Analytic Therapy (CAT)}

G. Yeates, ${ }^{1,2}$ M. Hamill, ${ }^{3}$ L. Sutton, ${ }^{3}$ K. Psaila, ${ }^{1}$ F. Gracey, ${ }^{1}$ S. Mohamed ${ }^{1}$ and J. O'Dell ${ }^{1}$

${ }^{1}$ Oliver Zangwill Centre for Neuropsychological Rehabilitation, Ely, Cambridge, United Kingdom

${ }^{2}$ Rehab UK, Birmingham, United Kingdom

${ }^{3}$ LHP Partnerships NHS Trust, Bury St Edmunds, Suffolk, United Kingdom

Common sequelae following frontal acquired brain injury (ABI) include

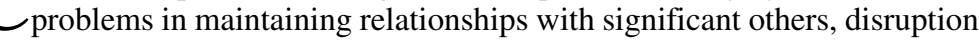
to self/identity and dysexecutive problems. The efficacy of psychotherapy interventions aimed at exploring psychosocial and relationship issues with these clients is in itself challenged by these problems. In particular, the therapeutic relationship is subject to dysexecutive influences. A working clinical formulation of interpersonal and identity problems in the context of dysexecutive impairment is suggested. This is achieved via integrating a neuropsychological model of goal-directed behaviour with a form of interpersonally-focused brief psychotherapy (Cognitive Analytic Therapy, CAT). That is, the interpersonal goal-directed sequence specified in a CAT formulation is adapted to include the neuropsychological difficulties in initiating, 
implementing and monitoring plans associated with frontal ABI, and linking these back to simultaneous interpersonal and identity outcomes. The use of cueing, electronic alerting and other compensatory interventions is specified within this formulation. Two case studies are presented for illustrative purposes, including one systematic therapy evaluation detailing shifts in identity and gains on measures of anxiety, anger and relationship perception as a result of the CAT intervention. Furthermore, the specific influence of alerting technology in this client's home was identified over and above the sharing of a clinical formulation and in-session techniques. Conclusions include the need to integrate cognitive rehabilitation techniques with psychological therapy approaches to optimise efficacy in ABI populations, and the need to formulate the interpersonal implications of introducing strategies such as alerting for individual clients. 